\title{
Viscosity and scale invariance in the unitary Fermi gas
}

\author{
Tilman Enss ${ }^{\mathrm{a}}$, Rudolf Haussmann ${ }^{\mathrm{b}}$, Wilhelm Zwerger ${ }^{\mathrm{a}}$ \\ ${ }^{a}$ Technische Universität München, James-Franck-Straße, D-85747 Garching, Germany \\ ${ }^{b}$ Fachbereich Physik, Universität Konstanz, D-78457 Konstanz, Germany
}

\begin{abstract}
We compute the shear viscosity of the unitary Fermi gas above the superfluid transition temperature, using a diagrammatic technique that starts from the exact Kubo formula. The formalism obeys a Ward identity associated with scale invariance which guarantees that the bulk viscosity vanishes identically. For the shear viscosity, vertex corrections and the associated Aslamazov-Larkin contributions are shown to be crucial to reproduce the full Boltzmann equation result in the high-temperature, low fugacity limit. The frequency dependent shear viscosity $\eta(\omega)$ exhibits a Drude-like transport peak and a power-law tail at large frequencies which is proportional to the Tan contact. The weight in the transport peak is given by the equilibrium pressure, in agreement with a sum rule due to Taylor and Randeria. Near the superfluid transition the peak width is of the order of $0.5 T_{F}$, thus invalidating a quasiparticle description. The ratio $\eta / s$ between the static shear viscosity and the entropy density exhibits a minimum near the superfluid transition temperature whose value is larger than the string theory bound $\hbar /\left(4 \pi k_{B}\right)$ by a factor of about seven.
\end{abstract}

PACS: 67.10.Jn; 67.85.Lm; 11.30.-j

\section{Introduction}

The remarkable derivation of a simple proportionality between the shear viscosity $\eta$ and the entropy per volume $s$ in a $\mathcal{N}=4$ supersymmetric Yang-Mills theory in the limit of infinite 't Hooft coupling $\lambda=g^{2} N \rightarrow \infty$ by Policastro, Son and Starinets [1] and the conjecture by Kovtun, Son and Starinets (KSS) [2] that the ratio $\eta / s$ is larger than the value $\hbar /\left(4 \pi k_{B}\right)$ found in this limit for all scale invariant, relativistic field theories have motivated the search for the 'perfect fluid' which realizes, or at least comes close to, this bound [3]. In spite of some theoretical counter-examples [4, 5, 6, 7, 8], the KSS conjecture turns out to be valid for all real fluids that are known. In particular, since the velocity of light does not appear in the KSS bound, the conjecture is applicable to both relativistic and non-relativistic field theories and may even be extended to complex, classical fluids. In the case of water, for instance, the minimum value for the ratio $\eta / s$ is found close to its critical point at $650 \mathrm{~K}$ and is only a factor of 25 above the KSS bound [2, 3]. Clearly, $\hbar$ is irrelevant for the minimum value of $\eta / s$ of water at these temperatures. Yet, as shown in Appendix A, there is a simple argument which shows that the viscosity minimum of purely classical fluids is not far above that expected from the KSS bound. This begs the question what are the necessary conditions for a fluid to be 'perfect' in the sense 
of a minimum value of $\eta / s$ that is limited only by quantum mechanics and, moreover, what is the role of scale invariance in this context? As pointed out by Kovtun, Son and Starinets [2] and discussed in more detail in a number of recent lecture notes [9, 10, 11] on connections between holographic duality and many-body physics, a crucial requirement for a fluid to come close to the KSS bound is the fact that it is strongly interacting and thus has no well-defined quasiparticles. Indeed, in a situation with proper quasiparticles, transport coefficients like the shear viscosity can be computed using kinetic theory. Since the lifetime broadening $\hbar / \tau$ is much less than the average energy $k_{B} T$ for well-defined quasiparticles, the resulting $\eta / s \gg \hbar / k_{B}$ is typically far above the KSS bound [3]. By contrast, for strongly coupled quantum field theories, the relaxation times are expected to be of order $\hbar /\left(k_{B} T\right)$. Since $\eta / s T$ is a characteristic time scale for shear relaxation, this immediately implies that $\eta / s$ is of order $\hbar / k_{B}$ in the strongly coupled limit. A nontrivial example in this context is the standard $S U(3)$ Yang-Mills theory. The associated $\eta / s$ ratio for the pure gauge theory has been calculated numerically using lattice QCD $[12,13,14]$. Its minimum appears near the deconfinement transition temperature and turns out to be rather close to the KSS bound. The standard Yang-Mills theory near $T_{c}$ and its supersymmetric extension - for which $\eta / s$ is independent of temperature and no welldefined quasiparticles exist at arbitrary energies - thus have very similar values of $\eta / s$. Adding fermions to the pure gauge theory, the ratio $\eta / s$ becomes an experimentally accessible quantity in high-energy, non-central collisions of heavy nuclei. The observed ratio $\eta / s$ of the quark-gluon plasma is around $0.4 \hbar / k_{B}$, i.e., a factor five above the KSS bound [3].

In a condensed matter context, generic examples for strongly coupled, finite temperature field theories for which no quasiparticle description holds are provided by models that exhibit a zero temperature critical point [15]. These systems are scale invariant at a critical value $g_{c}$ of the coupling $g$. At finite temperature $T$, there is a quantum critical regime above the critical point, which covers a finite window $T>\left|g-g_{c}\right|^{z \nu}$. In this regime, the thermal energy $k_{B} T$ is the only energy scale and correlations of the order parameter exhibit incoherent relaxation with a characteristic time scale $\tau_{\Psi}=\mathcal{C} \hbar /\left(k_{B} T\right)$ [11]. Here, $\mathcal{C}$ is a constant that only depends on the universality class of the quantum phase transition. In addition, universal behavior shows up in transport coefficients like the conductivity and also the shear viscosity in the hydrodynamic regime $\hbar \omega \ll k_{B} T$. A concrete example is the pseudo-relativistic theory of graphene where the ratio $\eta / s=\Phi_{\eta} \hbar / k_{B}$ has recently been calculated within a Boltzmann equation approach. The marginally irrelevant Coulomb interaction in this case gives rise to a logarithmic temperature dependence $\Phi_{\eta}=0.008 \ln ^{2}(1 / T)$ of the prefactor $\Phi_{\eta}$ [16]. This yields a monotonically increasing viscosity as the temperature approaches zero within the quantum critical regime. Logarithmic singularities as $T \rightarrow 0$ are also present in standard 2d Fermi liquids [17].

Here, we consider the shear viscosity for the unitary Fermi gas, a system of attractively interacting Fermions at infinite scattering length. The unitary Fermi gas is realized experimentally with ultracold atoms in a balanced mixture of, e.g., the two lowest hyperfine levels of ${ }^{6} \mathrm{Li}$ at a Feshbach resonance and has been studied quite extensively over the past few years $[18,19,20]$. It provides an example of a non-relativistic field theory that is both scale- and conformally invariant [21]. The underlying quantum critical point in this case is the zero density gas at unitarity, as was shown by Nikolić and Sachdev [22]. As a consequence of scale invariance, pressure $p$ and energy density $\epsilon$ of the gas are related by $p=2 \epsilon / 3$ [23]. Moreover, the bulk viscosity vanishes at all temperatures [24, 25]. For 
the shear viscosity, quantitative results so far are only available in the high-temperature limit $T \gg T_{F}$ where a classical description in terms of a Boltzmann equation is possible [26], and also deep in the superfluid regime $T \ll T_{c} \simeq 0.15 T_{F}$. In the superfluid, a finite viscosity arises from phonon-phonon collisions in the normal fluid component, giving rise to a rapid increase $\eta(T) \sim T^{-5}$ of the viscosity as the temperature approaches zero [27]. Since $\eta(T) \sim T^{3 / 2}$ also increases in the classical limit, both the viscosity and the ratio $\eta / s$ of the unitary gas necessarily exhibit a minimum, a behavior which is in fact typical for any fluid [3]. The major question which will be discussed in this paper, is where this minimum appears and what the associated value of the viscosity and entropy is. It turns out that the minimum in $\eta / s$ for the non-relativistic unitary gas is about a factor of seven above the KSS bound, rather close to the value that is found for the relativistic quark-gluon plasma. We also show that the minimum in $\eta$ implies a lower bound on the shear diffusion constant $D_{\eta}$, which is about $0.5 \hbar / \mathrm{m}$.

In detail, we determine the real part $\eta(\omega)$ of the frequency-dependent shear viscosity from a diagrammatic method that evaluates the stress tensor correlation function in the exact Kubo formula. Within a conserving approximation that respects all symmetries and the associated conservation laws, we obtain $\eta(\omega)$ in the normal phase and, in particular, the static viscosity to entropy density ratio $\eta(\omega=0) / s$. Our results for $\eta(\omega)$ show a Drude-like transport peak around $\omega=0$. Its width defines a viscous scattering rate $1 / \tau_{\eta}$ which obeys $\hbar / \tau_{\eta} \ll k_{B} T$ at high temperatures, where kinetic theory is applicable. Near the transition temperature $T_{c} \simeq 0.15 T_{F}$ to the superfluid phase of the unitary gas, the width approaches $0.5 k_{B} T_{F}$, thus clearly invalidating a quasiparticle description of viscous transport in this regime. The weight $W=2 \epsilon / 3=p$ of the Drude peak turns out to be equal to the pressure at all temperatures, consistent with a sum rule derived recently by Taylor and Randeria [28]. For large frequencies $\hbar \omega \gtrsim k_{B} T$, there is a crossover from the Drude peak to an inverse square-root tail $\eta(\omega) \sim 1 / \sqrt{\omega}$, whose amplitude is proportional to the Tan contact density $C$ [29]. In the high-temperature limit $T \gg T_{F}$ we complement our numerical solution of the transport integral equations by an analytical solution to leading order in the fugacity $z=e^{\beta \mu}$. We thus confirm that $(i)$ the Kubo formula yields exactly the same expression as the Boltzmann equation in this limit, and (ii) the vertex corrections, in particular the Aslamazov-Larkin contributions, are crucial even in the classical limit and increase the scattering time by a factor of almost three. This resolves an inconsistency between the Boltzmann equation result and a previous diagrammatic calculation in the high-temperature limit by Bruun and Smith [26]. Finally, we derive Ward identities associated with the scale invariance of the unitary gas and show that they are obeyed within our approximation for the bulk viscosity vertices at all frequencies and temperatures. As a consequence, the bulk viscosity vanishes identically $\zeta(\omega) \equiv 0$ $[24,21,28]$.

The paper is organized as follows: in section 2 we define the microscopic model for the unitary gas and discuss its scale invariance. The basic correlation functions that allow to calculate the shear viscosity from first principles via a Kubo formula are defined in section 3 together with a discussion of hydrodynamic relations and sum rules. In section 4 we give a qualitative discussion of the temperature dependence of the shear viscosity in the classical and in the deep superfluid limit, as well as near the superfluid transition temperature. The transport integral equations and their solution within a self-consistent Luttinger-Ward formalism are derived in section 5. An exact solution of these equations to leading order in the fugacity is presented in section 6 , which turns out to reproduce 
the full solution of the Boltzmann equation in the high temperature limit. In section 7 we discuss our results for the frequency dependent viscosity in the low temperaturebut still normal fluid - regime of the unitary gas, where the assumption of independent collisions of well defined quasiparticles that underlies the Boltzmann equation breaks down. In particular, we calculate the shear viscosity to entropy density ratio $\eta / s$, which displays a minimum value slightly above the transition to the superfluid. Conclusions and open questions are presented in section 8. There are three appendices: in Appendix A, we give an argument based on purely dimensional analysis which explains why the inherently quantum mechanical KSS bound applies even to purely classical systems like a Lennard-Jones fluid. Appendix B is devoted to the derivation of the Ward identities that result from scale invariance and the associated vanishing of the bulk viscosity. Finally, in Appendix $\mathrm{C}$ we analytically compute the tail coefficient of the shear viscosity at large frequencies.

\section{The unitary Fermi gas and scale invariance}

The basic model Hamiltonian that describes a two-component Fermi gas with contact interactions of strength $\bar{g}$ is given by

$$
\hat{H}=\sum_{\boldsymbol{p} \sigma} \varepsilon_{\boldsymbol{p}} c_{\boldsymbol{p} \sigma}^{\dagger} c_{\boldsymbol{p} \sigma}+\bar{g}(\Lambda) \sum_{\boldsymbol{p} \boldsymbol{p}^{\prime} \boldsymbol{q}} c_{\boldsymbol{p}+\boldsymbol{q} \uparrow}^{\dagger} c_{\boldsymbol{p}^{\prime}-\boldsymbol{q} \downarrow}^{\dagger} c_{\boldsymbol{p}^{\prime} \downarrow} c_{\boldsymbol{p} \uparrow}
$$

Here $\varepsilon_{\boldsymbol{p}}=\boldsymbol{p}^{2} / 2 \mathrm{~m}$ is the kinetic energy, while $\sigma=\uparrow, \downarrow$ denotes the two fermion species (hyperfine levels in the context of cold atoms). Since a contact interaction in three dimensions needs to be regularized, the coupling strength $\bar{g}(\Lambda)$ is cutoff-dependent. It is related to the renormalized physical coupling strength $g=4 \pi \hbar^{2} a / m$ that is fixed by the scattering length $a$ via $\bar{g}(\Lambda)=g /(1-2 a \Lambda / \pi)$. The unitary gas corresponds to the special case $g=\infty$ of infinite scattering length. In order to see that the system is scale invariant at this point, it is convenient to model the contact interaction by an exchange of bosons. In a path integral language, the partition function at temperature $T \equiv 1 / \beta$ can then be written as a double integral

$$
Z=\int \mathcal{D}\left(\bar{\psi}_{\sigma}, \psi_{\sigma}\right) \mathcal{D}\left(\bar{\psi}_{B}, \psi_{B}\right) e^{-S}
$$

over Grassmann fields $\bar{\psi}_{\sigma}, \psi_{\sigma}$ with $\sigma=\uparrow, \downarrow$ and a complex scalar field $\bar{\psi}_{B}, \psi_{B}$. In the case of a Feshbach resonance, this field has a direct physical interpretation as a bound fermion pair in the closed channel. The action that describes the interacting Fermi gas in this two-channel description is

$$
\begin{array}{r}
S=\int_{0}^{\beta} d \tau \int d^{3} x\left[\sum_{\sigma=\uparrow, \downarrow} \bar{\psi}_{\sigma}\left[\hbar \partial_{\tau}-\frac{\hbar^{2} \nabla^{2}}{2 m}\right] \psi_{\sigma}+\bar{\psi}_{B}\left[\hbar \partial_{\tau}-\frac{\hbar^{2} \nabla^{2}}{4 m}+\nu\right] \psi_{B}\right. \\
\left.+\tilde{g}\left(\bar{\psi}_{B} \psi_{\uparrow} \psi_{\downarrow}+\text { h.c. }\right)\right] .
\end{array}
$$

Here, $\nu$ is the detuning of the bosonic field, which allows to change the induced interaction $g \sim-\tilde{g}^{2} / \nu$ between the fermions. In particular, if $\tilde{g}$ is held at the fixed 
point corresponding to zero effective range, $g=\infty$ is reached at resonance $\nu \equiv 0$, where the bosonic field is massless. As was shown by Nikolić and Sachdev [22], the action $S\left[\psi_{\sigma}, \psi_{B}\right]$ at this point is invariant under scale transformations $\boldsymbol{x} \rightarrow \lambda \boldsymbol{x}$ and $\tau \rightarrow \lambda^{2} \tau$. The fermionic field $\psi_{\sigma}(\boldsymbol{x}, \tau) \rightarrow \lambda^{-3 / 2} \psi_{\sigma}\left(\lambda \boldsymbol{x}, \lambda^{2} \tau\right)$ transforms with the canonical scaling dimension, while the bosonic field $\psi_{B}(\boldsymbol{x}, \tau) \rightarrow \lambda^{-2} \psi_{B}\left(\lambda \boldsymbol{x}, \lambda^{2} \tau\right)$ acquires an anomalous dimension. As a result, the dynamics of the bosons, which arises from the second term in equation (3), is irrelevant at unitarity. The description of a finite fermion density requires adding a contribution $\mu \bar{\psi}_{\sigma} \psi_{\sigma}$ to the action. With a fixed value of the chemical potential $\mu$, this term explicitly breaks scale invariance. For the unitary gas, however, the relation $\mu \sim \xi(0) k_{F}^{2}$ between the chemical potential and the Fermi wave vector $k_{F}$ is identical to that of an ideal Fermi gas, except for a universal constant $\xi(0) \simeq 0.36-0.4$ that describes the lowering of the Fermi energy due to the attractive interactions [19, 20]. A scale transformation thus leaves the action invariant provided $\mu \rightarrow \lambda^{-2} \mu$ is changed accordingly to account for the change in density. Alternatively, one may introduce the chemical potential through a special choice for the (common) phase of the fields $\psi_{\sigma}$ and $\psi_{B}$ [30]. Scale invariance gives rise to a conserved dilatation current [21] which implies the relation $\epsilon=3 p / 2$ and the vanishing of the bulk viscosity: during a uniform expansion the system remains self-similar and no entropy is produced $[25,24]$. In fact, the bulk viscosity of the unitary gas vanishes for all frequencies [28], a result that will be derived from an exact Ward identity in Appendix B. A subtle point in this context is the issue of whether scale invariance of the action will be present in the full theory, i.e., whether the classical symmetry of $S$ survives quantum fluctuations [31, 32]. For the unitary gas with equal masses of the two spin components, which is the situation discussed in this work, this is believed to be the case. For different masses, however, scale invariance will be broken due to the appearance of an Efimov effect, i.e., three- and four-body bound states appear even in the absence of a two-body bound state [33, 34]. In particular, four-body bound states appear for mass ratios larger than a critical value 13.384 [35]. The breaking of scale invariance through the appearance of an Efimov effect has been discussed so far mostly for bosons [36] and leads to limit cycle flows in a renormalization group treatment [37].

\section{Kubo formula for the viscosity}

The unitary Fermi gas is in a fluid state at arbitrary temperatures and thus has well defined bulk and shear viscosities $\zeta$ and $\eta$. On a microscopic level, the viscosities are the zero frequency limits of linear response functions that may be calculated from first principles by the Kubo formula. Specifically, both the shear and bulk viscosities follow from the retarded correlation functions of the stress tensor $\Pi_{i j}$ (in the zero external momentum limit),

$$
\chi_{i j, k l}^{\mathrm{ret}}(\boldsymbol{q}=0, \omega)=i \int d t d^{3} x e^{i \omega t} \theta(t)\left\langle\left[\Pi_{i j}(\boldsymbol{x}, t), \Pi_{k l}(\mathbf{0}, 0)\right]\right\rangle,
$$

and its imaginary parts $\operatorname{Im} \chi_{i j, k l}^{\text {ret }}(\omega)$, which are odd functions of $\omega$. In particular, the real part of the frequency dependent shear viscosity is determined by the associated positive 
and even spectral function

$$
\operatorname{Re} \eta(\omega)=\frac{\operatorname{Im} \chi_{x y, x y}^{\mathrm{ret}}(\omega)}{\omega}
$$

and its static limit $\eta=\lim _{\omega \rightarrow 0} \operatorname{Re} \eta(\omega)$. A completely analogous expression exists for the bulk viscosity $\zeta(\omega)$ which involves the trace $\Pi_{i i}$ of the stress tensor,

$$
\operatorname{Re} \zeta(\omega)=\frac{\operatorname{Im} \chi_{i i, j j}^{\mathrm{ret}}(\omega)}{9 \omega}
$$

The microscopic expression for the stress tensor operator that enters equation (4) for a non-relativistic quantum many-body system has been derived by Martin and Schwinger [38]. In the case of a scale-invariant short-range potential, where $-r \partial_{r} V(r)=2 V(r)$, it is given by

$$
\Pi_{i j}(\boldsymbol{q}=0, t)=\frac{\hbar^{2}}{m} \sum_{\boldsymbol{k} \sigma} k_{i} k_{j} c_{\boldsymbol{k} \sigma}^{\dagger} c_{\boldsymbol{k} \sigma}+\int d^{3} x \int d^{3} r \frac{r_{i} r_{j}}{r^{2}} 2 V(\boldsymbol{r}): n_{\uparrow}(\boldsymbol{x}) n_{\downarrow}(\boldsymbol{x}+\boldsymbol{r}): .
$$

The second term explicitly involves the interaction and guarantees that the stress tensor satisfies local momentum current conservation as an operator equation (see appendix $\mathrm{B}$ in Ref. [21]). In the zero-frequency limit, the kinetic part of the stress tensor (7) is the one that appears in the viscous terms of the hydrodynamic equations [3], and also as a source term in the Boltzmann equation [39]. In general, the two contributions to the stress tensor (7) describe physically different processes for momentum relaxation upon insertion into the Kubo formula (4). In particular, the correlation function of two interaction contributions describes collisional transport due to interparticle forces, which is in fact the dominant contribution in the liquid phase [40]. By contrast, the kinetic part is associated with the transfer of transverse momentum due to free particle motion and dominates in the gaseous phase. As will be shown in section 5 , for the unitary gas the interaction part of the stress tensor is important to guarantee that the bulk viscosity vanishes, however it gives no contribution to the shear viscosity due to the zero-range nature of the interaction.

Quite generally, momentum current conservation implies that the stress tensor correlation functions are directly related to the current correlation functions, which are defined by

$$
\chi_{i, k}^{\mathrm{ret}}(\boldsymbol{q}, \omega)=i \int d t d^{3} x e^{i(\omega t-\boldsymbol{q x})} \theta(t)\left\langle\left[j_{i}(\boldsymbol{x}, t), j_{k}(\mathbf{0}, 0)\right]\right\rangle
$$

with

$$
j_{i}(\boldsymbol{q})=\sum_{\boldsymbol{k} \sigma} \hbar k_{i} c_{\boldsymbol{k}-\boldsymbol{q} / 2, \sigma}^{\dagger} c_{\boldsymbol{k}+\boldsymbol{q} / 2, \sigma}
$$

the current operator. In the limit $\boldsymbol{q}, \omega \rightarrow 0$, these correlation functions acquire a simple form, dictated by hydrodynamics. In particular, the existence of a finite shear viscosity implies diffusive relaxation of the transverse currents [41, 42]. The transverse part of the current correlation spectral function thus has the generic form (for small $\boldsymbol{q}, \omega$ )

$$
\frac{\operatorname{Im} \chi_{\perp}^{\mathrm{ret}}(\boldsymbol{q}, \omega)}{\omega}=\frac{\eta q^{2}}{\omega^{2}+\left(D_{\eta} q^{2}\right)^{2}}
$$


with a diffusion constant $D_{\eta}$ that is directly proportional to the shear viscosity. Indeed, the sum rule

$$
\lim _{\boldsymbol{q} \rightarrow 0} \int \frac{d \omega}{\pi} \frac{\operatorname{Im} \chi_{\perp}^{\mathrm{ret}}(\boldsymbol{q}, \omega)}{\omega}=\rho_{n}
$$

which quite generally defines the normal fluid density $\rho_{n}[41,42]$ immediately implies an Einstein relation

$$
\eta=\rho_{n} D_{\eta}
$$

between the shear viscosity and the associated diffusion constant that was first derived by Hohenberg and Martin [41]. The shear viscosity can thus be defined both from the stress tensor correlation function and, alternatively, from the diffusion constant that appears in the transverse current response [42, 28]. For non-superfluid systems, the diffusion constant $D_{\eta}$ is identical to the standard kinematic viscosity $\nu=\eta / \rho$.

For strongly interacting fluids, an exact calculation of correlation functions like the ones in equation (4) is hardly possible. It is therefore of considerable interest to find constraints that allow to check the validity of approximate calculations. Such constraints are derived generically from a short-time expansion of the correlation functions that can be expressed in terms of equilibrium expectation values of certain commutators. For the viscosities of a Fermi gas with contact interactions, this has been achieved recently by Taylor and Randeria [28]. As will be shown in section 5 below, a straightforward derivation of the relevant sum rule can be given using a Ward identity due to Polyakov [43], who has discussed the behavior of the shear viscosity near the critical point of a neutral superfluid within a microscopic approach. This Ward identity, which follows from momentum conservation, implies that the frequency-dependent shear viscosity at arbitrary values of the scattering length $a$ obeys the sum rule

$$
\frac{2}{\pi} \int_{0}^{\infty} d \omega\left[\eta(\omega)-\frac{\hbar^{3 / 2} C}{15 \pi \sqrt{m \omega}}\right]=\frac{2 \varepsilon}{3}-\frac{\hbar^{2} C}{6 \pi m a}=p-\frac{\hbar^{2} C}{4 \pi m a} .
$$

Here, in the second form of the equality, we have used the non-trivial relation between energy density and pressure away from unitarity, first derived by Tan [44]. The sum rule (13) has precisely the form given by Taylor and Randeria [28], however their highfrequency tail coefficient $C_{\eta}=C /(10 \pi)$ is larger by a factor of $3 / 2$ and there is also a difference in the prefactor of the contribution proportional to $C / a$ away from unitarity. The sum rule implies that $\eta(\omega)$ decays like $1 / \sqrt{\omega}$ at large frequencies with a prefactor that is determined by the Tan contact density $C$, which is a measure for two fermions with opposite spin to be close together $[29,44,45,46]$. Moreover, the area under the frequency-dependent viscosity of the unitary gas is fixed by the equilibrium pressure, since the $a^{-1}$ term is absent at unitarity. Both features are verified with high accuracy by our calculations of $\eta(\omega)$ that are detailed in section 7 .

\section{Temperature dependence of the shear viscosity}

For the unitary gas, the density $n=k_{F}^{3} /\left(3 \pi^{2}\right)$ fixes both the momentum and energy scale, which are the Fermi wavevector $k_{F}$ and the associated Fermi energy $\varepsilon_{F}=k_{B} T_{F}=$ 
$\hbar^{2} k_{F}^{2} /(2 m)$. Since the viscosity has dimensions $\hbar n$, purely dimensional arguments require the static shear viscosity to be of the form

$$
\eta(T)=\hbar n \alpha(\theta)
$$

where $\theta=T / T_{F}$ is the dimensionless temperature scale and $\alpha(\theta)$ a universal scaling function. Remarkably, this function is fixed up to a universal constant $\mathcal{O}(1)$ in the high temperature limit. This is based on the counter-intuitive fact that the viscosity of a classical gas is independent of its density [47]. Since $T_{F} \sim n^{2 / 3}$, this requires $\alpha(\theta \gg 1) \sim \theta^{3 / 2}$, i.e., a shear viscosity that increases like $T^{3 / 2}$. The same qualitative result is obtained from the kinetic theory expression $\eta(T) \simeq\langle p\rangle / \sigma(T)$ with $\langle p\rangle$ an average momentum and $\sigma(T)$ a thermally averaged cross section. Since the differential cross section for a collision with a given relative momentum $p$ is $d \sigma / d \Omega=\hbar^{2} / p^{2}$ at unitarity, this immediately gives $\eta \simeq p_{T}^{3} / \hbar^{2}$ with $p_{T}=\sqrt{2 \pi m k_{B} T}$ the thermal momentum. Note that $\eta$ scales like $1 / \hbar^{2}$ even in the classical limit, provided the assumption of zero range $\mathrm{s}$-wave scattering remains valid in this regime.

At low temperatures $\theta \lesssim 0.15$, the unitary Fermi gas is a superfluid. Contrary to naive expectations, a superfluid is not a 'perfect fluid' despite the fact that there is a vanishing viscosity here. In particular, superfluids do not provide trivial counterexamples to the KSS conjecture. Indeed, according to the Landau two-fluid picture, the superfluid component has both zero viscosity and zero entropy, so $\eta / s$ is undefined at $T=0$. At any finite temperature, however, a normal component appears, whose entropy and viscosity are non-zero. For the bosonic superfluid ${ }^{4} \mathrm{He}$ this was discussed already by Landau and Khalatnikov in 1949 [48]. In the low temperature, phonon-dominated regime, they found that the shear viscosity of the normal component grows like $T^{-5}$ because the mean free path for phonon-phonon collisions, which are necessary for the relaxation of shear, diverges. Specifically, with the assumption that the phonon dispersion has negative curvature and thus no Beliaev decay is possible (this assumption is actually violated in superfluid ${ }^{4} \mathrm{He}$ but is likely to hold for the unitary Fermi gas, see [49]), the calculations of Landau and Khalatnikov predict the low temperature shear viscosity to be

$$
\eta(T \rightarrow 0)=\rho_{n}(T) \frac{\rho^{2} c_{s}^{3}}{\hbar^{2}} \frac{2^{13}(2 \pi)^{7}}{9(13) !(u+1)^{4}}\left(\frac{\hbar c_{s}}{k_{B} T}\right)^{9} .
$$

Here $\rho_{n}(T)=\left(2 \pi^{2} \hbar / 45 c_{s}\right)\left(k_{B} T / \hbar c_{s}\right)^{4}$ is the normal fluid mass density and $u=d \ln c_{s} / d \ln n$ is the dimensionless strength of the non-linear corrections to the leading-order quantum hydrodynamic Hamiltonian which lead to phonon-phonon scattering. The viscosity of the normal fluid component thus asymptotically diverges like $T^{-5}$. As realized by Rupak and Schäfer [27] the same behavior is expected in the unitary Fermi gas. Indeed, at temperatures far below $T_{c}$, the microscopic nature of the superfluid is irrelevant and the linearly dispersing Bogoliubov-Anderson phonons are the only excitations that remain. As a result, equation (15) for the viscosity applies also to the unitary Fermi gas, provided the exact values of the sound velocity $c_{s}$ and coupling constant $u$ are inserted. At unitarity, the sound velocity $c_{s}=v_{F} \sqrt{\xi / 3} \simeq 0.36 v_{F}$ is directly proportional to the Fermi velocity $v_{F}$ with a factor that is determined by the universal Bertsch parameter $\xi \simeq 0.36-0.4$ [19]. Since $v_{F} \sim n^{1 / 3}$ as for an ideal Fermi gas, the dimensionless coupling constant that fixes the strength of the phonon-phonon scattering amplitude has the universal value $u=1 / 3$. Together with the standard low-temperature expression 
$s=2 \pi^{2} k_{B} / 45\left(k_{B} T / \hbar c_{s}\right)^{3}$ for the entropy density of a scalar phonon field, the viscosity to entropy density ratio

$$
\frac{\eta(T \rightarrow 0)}{s}=\frac{\hbar}{k_{B}} 2.15 \times 10^{-5} \xi^{5} \theta^{-8}
$$

of the unitary Fermi gas at temperatures far below the superfluid transition diverges rapidly as $\left(T_{F} / T\right)^{8}$. The associated prefactor agrees within $2 \%$ with that found from a diagrammatic calculation by Rupak and Schäfer [27], which is based on the next-toleading terms in the effective field theory of the unitary gas by Son and Wingate [30] (note the different prefactor of the entropy in [27]). It is a pure number that only contains the Bertsch parameter. Note that due to $\rho_{n}(T) / s(T)=T / c_{s}^{2}$ at very low temperatures, the ratio $\eta / s T=D_{\eta} / c_{s}^{2}$ is just the characteristic relaxation time $\tau_{\eta}$ for shear fluctuations. According to equation (15), this relaxation time diverges like $\tau_{\eta} \sim T^{-9}$ as $T \rightarrow 0$, much stronger than the pure thermal energy time scale $\hbar /\left(k_{B} T\right)$. In fact, the latter would lead to a viscosity to entropy density ratio which approaches a constant at very low temperatures, as in the $\mathcal{N}=4$ supersymmetric Yang-Mills theory, where no quasiparticles exist.

Near the superfluid transition temperature $T_{c}$, the universal scaling function $\alpha(\theta)$ will be continuous, yet there will be singularities in higher derivatives. Within the conventional theory of critical dynamics, the non-analytical behavior of $\eta=\rho_{n} D_{\eta}$ near $T_{c}$ only comes from the thermodynamic singularity in $\rho_{n}(T)$ [50]. Since $\rho_{n}(T)=\rho-c^{\prime} \theta(-t)|t|^{\nu}$ with $c^{\prime}$ a positive constant and $\nu \approx 0.67$ the universal critical exponent of the $3 \mathrm{D}$ XYmodel, the conventional theory predicts that $\alpha(\theta)$ reaches its finite value at $T_{c}$ with an infinite slope as the critical temperature is approached from below [51]. A non-analytical temperature dependence of the shear viscosity is indeed observed near the $\lambda$ point of superfluid ${ }^{4} \mathrm{He}$. In this case, however, a singularity of the form $\operatorname{sign}(t)|t|^{\omega}$ appears on both sides of the transition [52]. The associated exponent $\omega$ is consistent with the prediction $\omega=\nu$ of conventional theory below $T_{c}$, however it has a different value $\omega \approx 0.8$ above the critical temperature. This behavior can be explained within a semi-phenomenological approach [53] which is, however, genuine for a liquid state. Thus it cannot be carried over to the case of the unitary Fermi gas, for which the precise behavior of $\alpha(\theta)$ near the critical point remains an open problem.

\section{Diagrammatic evaluation of the stress tensor correlation functions}

In order to compute the stress correlation functions (4) we start from the singlechannel model and derive the corresponding stress tensor in the two-channel model. This has the advantage that the potential (fermion interaction) term of the stress tensor becomes simply a detuning (mass term) of the bosonic field in the two-channel model. We then derive the exact expression (30) for the stress correlation function in the twochannel model, including both kinetic and potential terms, in the zero-range limit. This expression is evaluated in the self-consistent T-matrix approximation $[54,55]$. In the two-channel model we compute the fermionic and bosonic self-energies self-consistently at the one-loop level but neglect the loop corrections to the Yukawa coupling between fermions and bosons which appear only at two-loop order (cf. Fig. 1). Variation of these coupled single-particle equations with respect to a time-dependent external field results 
in a set of transport equations for the viscosity response functions. A crucial feature of this procedure is that it respects the symmetries of the underlying model exactly, in particular it obeys scale invariance and thus leads to a vanishing bulk viscosity as required.

Since the continuum model is Galilean invariant, one may expand the stress tensor operator (7) of the single-channel model (1) into spherical harmonics with angularmomentum quantum number $\ell$ :

$$
\Pi_{\ell}=\sum_{\boldsymbol{p} \sigma} 2 \varepsilon_{p} Y_{\ell}(\hat{\boldsymbol{p}}) c_{\boldsymbol{p} \sigma}^{\dagger} c_{\boldsymbol{p} \sigma}+2 \delta_{\ell 0} \bar{g}(\Lambda) \int d^{3} r: n_{\uparrow}(\boldsymbol{r}) n_{\downarrow}(\boldsymbol{r}):
$$

where the spherical harmonics $Y_{\ell}(\hat{\boldsymbol{p}})$ depend on the angle of the vector $\boldsymbol{p}=(p, \theta, \phi)$ as $Y_{\ell=0}(\theta, \phi)=1$ and $Y_{\ell=2}(\theta, \phi)=\sin ^{2}(\theta) \sin (\phi) \cos (\phi)$. (For convenience we omit the standard normalization factor $1 / \sqrt{4 \pi}$ in the spherical harmonics.) For a quadratic dispersion the fermion kinetic term admits $\ell=0$ and $\ell=2$. Note that for the scale-invariant, zero-range model at unitarity the interaction term contributes only for $\ell=0$ !

In order to compute linear response one has to add a perturbation to the Hamiltonian which couples the stress tensor to a time-dependent external field,

$$
\Delta H(t)=\sum_{\ell} h_{\ell}(t) \Pi_{\ell}(t)=\sum_{\boldsymbol{p} \sigma \ell} 2 h_{\ell}(t) \varepsilon_{p} Y_{\ell}(\hat{\boldsymbol{p}}) c_{\boldsymbol{p} \sigma}^{\dagger} c_{\boldsymbol{p} \sigma}+2 h_{\ell=0}(t) \bar{g}(\Lambda) \int d^{3} r: n_{\uparrow}(\boldsymbol{r}) n_{\downarrow}(\boldsymbol{r}): .
$$

This amounts to the replacement $\varepsilon_{p} \mapsto \varepsilon_{p}\left[1+2 h_{\ell}(t)\right]$ and $\bar{g}(\Lambda) \mapsto \bar{g}(\Lambda)\left[1+2 h_{\ell}(t) \delta_{\ell 0}\right]$ in the full Hamiltonian, and likewise in the full action at unitarity

$$
\begin{aligned}
S[h]=\int_{0}^{\beta} d \tau\left[\sum_{\boldsymbol{p} \sigma}\left[1+\sum_{\ell} 2 h_{\ell}(\tau) Y_{\ell}(\hat{\boldsymbol{p}})\right] \varepsilon_{p} \bar{c}_{\boldsymbol{p} \sigma} c_{\boldsymbol{p} \sigma}\right. & +\tilde{g} \bar{\psi}_{B} \psi_{\uparrow} \psi_{\downarrow}+\text { h.c. } \\
& \left.-\frac{\tilde{g}^{2}}{\bar{g}(\Lambda)\left[1+2 h_{\ell=0}(\tau)\right]} \bar{\psi}_{B} \psi_{B}\right] .
\end{aligned}
$$

The change in the action due to the external field, $\Delta S=S[h]-S$, can be parametrized as

$$
\Delta S=\sum_{X X^{\prime}}\left[\sum_{\sigma} \bar{\psi}_{\sigma}(X) U_{\sigma, X X^{\prime}} \psi_{\sigma}\left(X^{\prime}\right)+\bar{\psi}_{B}(X) U_{B, X X^{\prime}} \psi_{B}\left(X^{\prime}\right)\right]
$$

with the coefficient functions

$$
\begin{aligned}
& U_{\sigma, X X^{\prime}}=\int d \tau \sum_{\ell} h_{\ell}(\tau) Y_{\ell}(\hat{\boldsymbol{p}}) T_{\sigma \ell}^{(0)}\left(\tau X X^{\prime}\right), \\
& U_{B, X X^{\prime}}=\tilde{g}^{2} \int d \tau \sum_{\ell} \frac{h_{\ell}(\tau)}{1+2 h_{\ell}(\tau)} S_{\ell}^{(0)}\left(\tau X X^{\prime}\right) .
\end{aligned}
$$

For convenience we use a short-hand notation $X=(\boldsymbol{r}, \tau)$ for the real-space argument and $K=\left(\boldsymbol{k}, i \epsilon_{n}\right)$ for the Fourier argument, and $\sum_{X}=\int d^{3} r \hbar^{-1} \int d \tau$ as well as $\delta_{X X^{\prime}}=$ 
$\delta\left(\boldsymbol{r}-\boldsymbol{r}^{\prime}\right) \hbar \delta\left(\tau-\tau^{\prime}\right)$. Equations (21), (22) define the bare fermionic and bosonic viscosity response vertices at unitarity,

$$
\begin{aligned}
T_{\sigma \ell}^{(0)}\left(\tau X_{1} X_{1}^{\prime}\right) & =\frac{\hbar^{2} \nabla_{1} \nabla_{1}^{\prime}}{m} \delta_{X_{1} X_{1}^{\prime}} \delta\left(\tau-\tau_{1}\right), \\
S_{\ell}^{(0)}\left(\tau X_{1} X_{1}^{\prime}\right) & = \begin{cases}2 \bar{g}(\Lambda)^{-1} \delta_{X_{1} X_{1}^{\prime}} \delta\left(\tau-\tau_{1}\right) & \text { for } \ell=0 \\
0 & \text { for } \ell=2 .\end{cases}
\end{aligned}
$$

The response of the grand potential to the external field in terms of the fermionic Green's functions $G_{\sigma, X X^{\prime}}=\left\langle\mathcal{T} \psi_{\sigma}(X) \psi_{\sigma}^{\dagger}\left(X^{\prime}\right)\right\rangle$ and bosonic Green's functions $G_{B, X X^{\prime}}=$ $\left\langle\mathcal{T} \psi_{B}(X) \psi_{B}^{\dagger}\left(X^{\prime}\right)\right\rangle$ is

$$
\begin{aligned}
\delta \Omega & =\operatorname{tr}\left[\left(\delta U_{\sigma}\right) G_{\sigma}\right]+\operatorname{tr}\left[\left(\delta U_{B}\right) G_{B}\right] \\
& =\operatorname{tr}\left[T_{\sigma \ell}^{(0)} G_{\sigma} \delta h_{\ell}(\tau)\right]-\operatorname{tr}\left[S_{\ell}^{(0)} \Gamma \frac{\delta h_{\ell}(\tau)}{\left(1+2 h_{\ell}(\tau)\right)^{2}}\right]
\end{aligned}
$$

where the trace includes the spin sum and in the second line additionally the $\ell$ sum. In the second line the bosonic Green's function is replaced by the vertex function $\Gamma_{X X^{\prime}}=$ $-\tilde{g}^{2} G_{B, X X^{\prime}}$. Hence, we obtain

$$
-\frac{\delta \Omega}{\delta h_{\ell}(\tau)}=-\sum_{\sigma X X^{\prime}} T_{\sigma \ell}^{(0)}\left(\tau X X^{\prime}\right) G_{\sigma, X^{\prime} X}+\frac{1}{\left(1+2 h_{\ell}(\tau)\right)^{2}} \sum_{X X^{\prime}} S_{\ell}^{(0)}\left(\tau X X^{\prime}\right) \Gamma_{X^{\prime} X} .
$$

In particular, for a static scaling perturbation $h_{\ell=0}(\tau) \equiv h$ we recover the Tan energy formula [29] with the correct UV regularization,

$$
3 p=\left\langle\Pi_{i i}\right\rangle=-\left.\frac{d \Omega}{d h}\right|_{h=0}=2 \sum_{\boldsymbol{k} \sigma} \frac{\hbar^{2} k^{2}}{2 m}\left(n_{k \sigma}-\frac{C}{k^{4}}\right)=2\langle H\rangle=2 \varepsilon,
$$

where the local limit of the vertex function $\left.\Gamma_{X X^{\prime}}\right|_{X^{\prime}=X^{+}}$has been expressed in terms of the contact density (C.4). The Kubo formula (4) in imaginary time can be re-expressed in partial-wave components as

$$
\chi_{\ell}(\tau)=\int d^{3} r\left\langle\mathcal{T} \Pi_{\ell}(\boldsymbol{r}, \tau) \Pi_{\ell}(\mathbf{0}, 0)\right\rangle,
$$

where $\mathcal{T}$ denotes time ordering. This stress correlation function can be obtained from the second derivative of the grand potential (27),

$$
\begin{aligned}
\chi_{\ell}(\tau) & =-\left.\frac{\delta^{2} \Omega}{\delta h_{\ell}(\tau) \delta h_{\ell}(0)}\right|_{h_{\ell}=0} \\
& =-\operatorname{tr}\left[T_{\sigma \ell}^{(0)}(0) \tilde{T}_{\sigma \ell}(\tau)\right]+\operatorname{tr}\left[S_{\ell}^{(0)}(0)\left(\tilde{S}_{\ell}(\tau)-4 \delta(\tau) \Gamma(0)\right)\right]
\end{aligned}
$$

where the last term in the square brackets comes from the explicit $h_{\ell}$ dependence in the second term of equation (27). Note also that this last term is crucial to obtain a vanishing bulk viscosity at unitarity $a^{-1}=0$, as we will show in Appendix B using 
the Ward identities that follow from scale invariance. $\tilde{T}_{\ell}$ and $\tilde{S}_{\ell}$ are the fermionic and bosonic viscosity response functions

$$
\begin{aligned}
& \tilde{T}_{\sigma \ell}\left(\tau X X^{\prime}\right)=\left.\frac{\delta G_{\sigma, X X^{\prime}}}{\delta h_{\ell}(\tau)}\right|_{h_{\ell}=0}=\left\langle\mathcal{T} \Pi_{\ell}(\tau) \psi_{\sigma}(X) \psi_{\sigma}^{\dagger}\left(X^{\prime}\right)\right\rangle \\
& \tilde{S}_{\ell}\left(\tau X X^{\prime}\right)=\left.\frac{\delta \Gamma_{X X^{\prime}}}{\delta h_{\ell}(\tau)}\right|_{h_{\ell}=0}=-\tilde{g}^{2}\left\langle\mathcal{T} \Pi_{\ell}(\tau) \psi_{B}(X) \psi_{B}^{\dagger}\left(X^{\prime}\right)\right\rangle .
\end{aligned}
$$

Note that for the case of the shear viscosity where $S_{\ell}^{(0)}=0$, the viscosity response function can be expressed by the $\mathrm{L}$ function [54] as $\tilde{T}_{\ell}=L T_{\ell}^{(0)}$, and the correlation function (30) reduces to the well-known form $\chi_{\ell=2}(\tau)=-\operatorname{tr}\left[T_{\sigma, \ell=2}^{(0)}(\tau) L T_{\sigma, \ell=2}^{(0)}(0)\right]$.

The formalism developed so far now allows to derive the sum rule given in equation (13), starting from the Ward identity for momentum conservation in the static limit of external $\omega=0, \boldsymbol{q} \rightarrow 0$ which reads [43, eq. (5.26)]

$$
\tilde{T}_{x y}=-p_{x} \partial G / \partial p_{y} .
$$

Here $\tilde{T}_{x y}=\tilde{T}_{\ell=2} Y_{\ell=2}$ is the vertex function of the stress tensor (cf. equation (31)). Starting from equation (30) for the shear viscosity correlation function, we insert the bare shear viscosity vertex $T_{\ell=2}^{(0)}\left(p, i \epsilon_{n}\right)=\hbar^{2} p^{2} / m$ from equation (23) and the full shear viscosity vertex $T_{\ell=2}^{(0)}\left(p, i \epsilon_{n}\right)=-p \partial G / \partial p$ from the Ward identity (33) and obtain at zero external Matsubara frequency $i \omega_{m}=0$ (note that $\chi_{x y, x y}\left(i \omega_{m}\right)=\chi_{\ell=2}\left(i \omega_{m}\right) / 15$ due to the angular average of $\left[Y_{\ell=2}\right]^{2}$ )

$$
\begin{aligned}
\chi_{x y, x y}\left(i \omega_{m}=0\right) & =-\frac{1}{15} \sum_{p \sigma} \frac{1}{\beta} \sum_{i \epsilon_{n}} T_{\ell=2}^{(0)}\left(p, i \epsilon_{n}\right) \tilde{T}_{\ell=2}\left(p, i \epsilon_{n}\right) \\
& =\frac{1}{15} \sum_{p \sigma} \frac{1}{\beta} \sum_{i \epsilon_{n}} \frac{\hbar^{2} p^{2}}{m} p \frac{\partial G\left(p, i \epsilon_{n}\right)}{\partial p} \\
& =-\frac{2}{15} \sum_{p \sigma} \varepsilon_{p} p \frac{\partial n_{p}}{\partial p}
\end{aligned}
$$

in terms of the fermionic momentum distribution $n_{p}=-\beta^{-1} \sum_{i \epsilon_{n}} G\left(p, i \epsilon_{n}\right)$. We now integrate by parts, with a boundary term at momentum cutoff $\Lambda$, and employ the Tan energy formula [29] to express the sum over the momentum distribution by the internal energy density $\varepsilon$,

$$
\begin{aligned}
\chi_{x y, x y}\left(i \omega_{m}=0\right) & =\frac{2}{15}\left[5 \sum_{p \sigma} \varepsilon_{p} n_{p}-\left.\frac{\hbar^{2} p^{5} n_{p}}{2 \pi^{2} m}\right|_{0} ^{\Lambda}\right] \\
& =\frac{2 \varepsilon}{3}+\frac{4 \hbar^{2} C \Lambda}{15 \pi^{2} m}-\frac{\hbar^{2} C}{6 \pi m a} .
\end{aligned}
$$

If the momentum cutoff $\Lambda$ is translated into a frequency cutoff $\Omega=\hbar \Lambda^{2} / m[28$, endnote 39 ] we arrive at the sum rule given in equation (13).

The derivation of the correlation function (30) so far has been completely general and exact. The remaining challenge then is to evaluate the viscosity response functions $\tilde{T}_{\ell}$ 

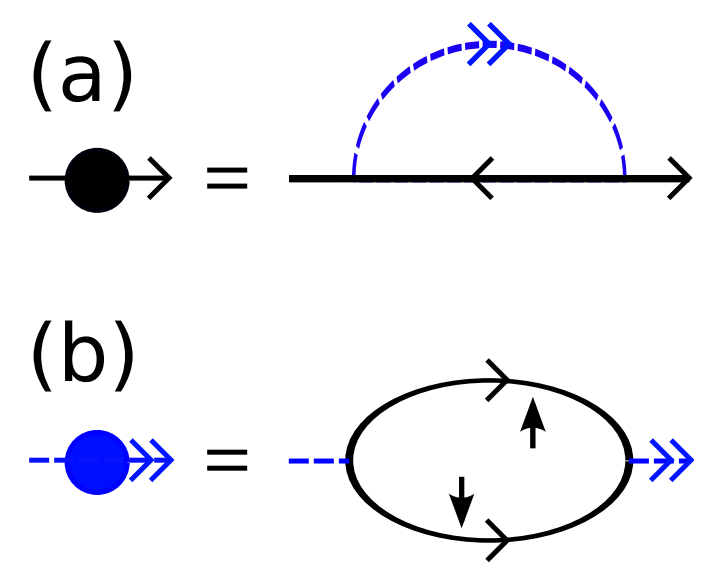

Figure 1: [color online] Diagrammatic contributions to the fermionic and bosonic self-energies.

and $\tilde{S}_{\ell}$ within the microscopic model for the unitary Fermi gas. In the following we shall do this within the T-matrix approximation. We start from the Dyson equation for the fermionic Green's function $G_{\sigma, X X^{\prime}}$ in the Matsubara formalism,

$$
G_{\sigma, X X^{\prime}}^{-1}=G_{0, \sigma X X^{\prime}}^{-1}-U_{\sigma, X X^{\prime}}-\Sigma_{\sigma, X X^{\prime}}
$$

with bare Green's functions $G_{0}(K)^{-1}=-\left(i \hbar \epsilon_{n}+\mu-\varepsilon_{\boldsymbol{k}}\right)$ and the external field $U_{\sigma, X X^{\prime}}$ from equation (21). In the T-matrix approximation the fermionic self-energy describes how fermions scatter off pair fluctuations (cf. Fig. 1a),

$$
\Sigma_{\sigma, X X^{\prime}}=G_{-\sigma, X^{\prime} X} \Gamma_{X X^{\prime}} .
$$

The Bethe-Salpeter equation for the vertex function which mediates the resonant FermiFermi interaction is

$$
\Gamma_{X X^{\prime}}^{-1}=\bar{g}(\Lambda)^{-1}-U_{B, X X^{\prime}}+G_{\uparrow, X X^{\prime}} G_{\downarrow, X X^{\prime}} .
$$

It contains the inverse bare coupling $\bar{g}(\Lambda)^{-1}=g^{-1}-m \Lambda /\left(2 \pi^{2} \hbar^{2}\right)$, the external field $U_{B}$ from equation (22), and the bosonic self-energy (cf. Fig. 1b). As mentioned above, the dynamics of the pair field at unitarity $a^{-1} \rightarrow 0$ only arises from the excitation of fermion pairs while the dynamics of the bosons is irrelevant. Since we consider a balanced gas with equal populations of fermion species $\mu_{\uparrow}=\mu_{\downarrow}=\mu$ we will henceforth drop the spin index $\sigma$.

The T-matrix approximation for the exact viscosity response vertices is then obtained by taking the derivative of the self-consistency equations (35)-(37) with respect to the external field $h_{\ell}(\tau)$ (cf. Fig. 2); in this way it is guaranteed that the conservation laws are satisfied. The amputated viscosity response vertex

$$
T_{\ell}\left(\tau X X^{\prime}\right)=-\frac{\delta G_{X X^{\prime}}^{-1}}{\delta h_{\ell}(\tau)}=\sum_{Y Y^{\prime}} G_{X Y}^{-1} \tilde{T}_{\ell}\left(\tau Y Y^{\prime}\right) G_{Y^{\prime} X^{\prime}}^{-1}
$$




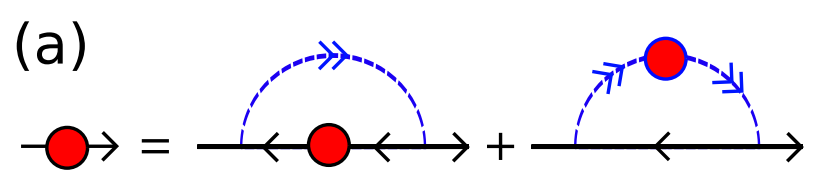

(b)

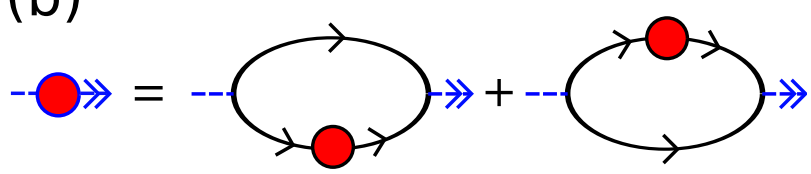

(c)

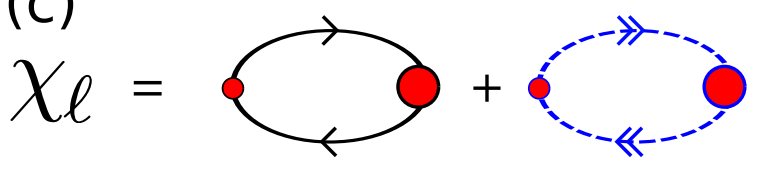

Figure 2: [color online] Diagrammatic contributions to the renormalized viscosity response functions $T_{\ell}$ and $S_{\ell}$. (a) The full fermionic response function $T_{\ell}$ has Maki-Thompson and Aslamazov-Larkin contributions, in addition to the bare vertex $T_{\ell}^{(0)}$. (b) The full bosonic response function $S_{\ell}$ has two contributions which are equivalent for equal fermion masses and populations, in addition to the bare vertex $S_{\ell}^{(0)}$. (c) The viscosity correlation function is given by a skeleton diagram with one bare and one renormalized viscosity response vertex and full propagators (fermionic and bosonic). In particular, the second diagram describes transport via collective modes.

is given by the variation of the Dyson equation (35) with respect to $h_{\ell}(\tau)$,

$$
T_{\ell}\left(\tau X X^{\prime}\right)=T_{\ell}^{(0)}\left(\tau X X^{\prime}\right)+T_{\ell}^{\mathrm{MT}}\left(\tau X X^{\prime}\right)+T_{\ell}^{\mathrm{AL}}\left(\tau X X^{\prime}\right)
$$

with the Maki-Thompson and Aslamazov-Larkin vertex corrections

$$
\begin{aligned}
& T_{\ell}^{\mathrm{MT}}\left(\tau X X^{\prime}\right)=\frac{\delta G_{X^{\prime} X}}{\delta h_{\ell}(\tau)} \Gamma_{X X^{\prime}}=\tilde{T}_{\ell}\left(\tau X^{\prime} X\right) \Gamma\left(X X^{\prime}\right), \\
& T_{\ell}^{\mathrm{AL}}\left(\tau X X^{\prime}\right)=G_{X^{\prime} X} \frac{\delta \Gamma_{X X^{\prime}}}{\delta h_{\ell}(\tau)}=G_{X^{\prime} X} \tilde{S}_{\ell}\left(\tau X X^{\prime}\right) .
\end{aligned}
$$

The amputated bosonic viscosity response vertex

$$
S_{\ell}\left(\tau X X^{\prime}\right)=-\frac{\delta \Gamma_{X X^{\prime}}^{-1}}{\delta h_{\ell}(\tau)}=\sum_{Y Y^{\prime}} \Gamma_{X Y}^{-1} \tilde{S}_{\ell}\left(\tau Y Y^{\prime}\right) \Gamma_{Y^{\prime} X^{\prime}}^{-1}
$$

is given by the derivative of the Bethe-Salpeter equation (37),

$$
S_{\ell}\left(\tau X X^{\prime}\right)=S_{\ell}^{(0)}\left(\tau X X^{\prime}\right)-2 G_{X X^{\prime}} \tilde{T}_{\ell}\left(\tau X X^{\prime}\right) .
$$

The bosonic viscosity vertex describes the response of the pair field to a scaling or shear perturbation. The bare bosonic viscosity vertex $S_{\ell}^{(0)}$ serves to remove the UV divergence of the particle-particle loop (second term) which is present only for the bulk viscosity $\ell=0$. 
The self-consistent transport equations for the two-channel model are equivalent to those of the standard T-matrix approximation in a purely fermionic description. Specifically, the two fermionic vertex corrections $T_{\ell}^{\mathrm{MT}}$ and $T_{\ell}^{\mathrm{AL}}$ above are precisely those arising in the self-consistent solution of the integral kernel of the $L$ function, as introduced by Baym [54, equation (60)]. In physical terms, the first term describes the interaction between two fermions by exchange of a single pair while in the second term two pairs are exchanged. In the context of calculating the change in conductivity due to superconducting fluctuations, these terms are called the Maki-Thompson (MT) and Aslamazov-Larkin (AL) contributions respectively [56], a notation that will be used also in the present context. Note that the second term explicitly includes particle-hole fluctuations, and it is also referred to as the "box diagram" in a functional renormalization group approach to the thermodynamics of the unitary Fermi gas [57]. These vertex corrections are in fact crucial to obtain an approximation which satisfies the conservation laws of the underlying model.

The self-consistent equations (35)-(43) have a structure similar to the equations of the Luttinger-Ward approach to the BCS-BEC crossover developed in our previous work $[58,59]$. In particular, using Fourier transforms and the convolution theorem they become algebraic equations which afford an efficient numerical solution. The numerical calculations are performed in three steps. In the first step the Green's function $G_{X X^{\prime}}$ and the vertex function $\Gamma_{X X^{\prime}}$ are calculated by solving the self-consistent equations (35)(37) iteratively. Without the external fields $U_{\sigma}$ and $U_{B}$ the Dyson and Bethe-Salpeter equations are diagonal in Fourier space, while the fermionic and bosonic self-energies are local in real space. Hence, the coupled equations are solved efficiently by going back and forth between real and Fourier space.

In the second step $G_{X X^{\prime}}$ and $\Gamma_{X X^{\prime}}$ are used as input for the self-consistent equations (38)-(43) to calculate the viscosity response functions $\tilde{T}_{\ell}, \tilde{S}_{\ell}$. Again, the integral equations (38) and (42) become algebraic and are solved in Fourier space, while the other equations remain local in real space. Note that the spatial Fourier transform between radial distances $r$ and radial wavenumber $k$ for the partial-wave component $\ell$ is given by

$$
\begin{aligned}
& T_{\ell}(k)=4 \pi(-i)^{\ell} \int_{0}^{\infty} d r r^{2} j_{\ell}(k r) T_{\ell}(r), \\
& T_{\ell}(r)=\frac{i^{\ell}}{2 \pi^{2}} \int_{0}^{\infty} d k k^{2} j_{\ell}(k r) T_{\ell}(k) .
\end{aligned}
$$

In the third step the correlation function $\chi_{\ell}\left(i \omega_{m}\right)$ is computed from $(30)$. It is continued analytically from the discrete imaginary Matsubara frequencies $i \omega_{m}$ to the continuous real frequencies $\omega$ via both the Padé method and a model fit function (cf. section 7 ). We thus obtain the retarded correlation function $\chi_{\ell}^{\text {ret }}(\omega)=\chi_{\ell}^{\prime}(\omega)+i \chi_{\ell}^{\prime \prime}(\omega)$. Finally, the real parts of the viscosities $\eta$ and $\zeta$ are obtained from the correlation functions for $\ell=2$ and $\ell=0$ according to (cf. equations (5) and (6))

$$
\begin{aligned}
& \operatorname{Re} \eta(\omega)=\frac{\operatorname{Im} \chi_{\ell=2}^{\mathrm{ret}}(\omega)}{15 \omega}, \\
& \operatorname{Re} \zeta(\omega)=\frac{\operatorname{Im} \chi_{\ell=0}^{\mathrm{ret}}(\omega)}{9 \omega},
\end{aligned}
$$

where the prefactor of $\eta$ comes from the angular integration of the spherical harmonics $\left[Y_{\ell=2}(\hat{\boldsymbol{p}})\right]^{2}$. Alternatively, one may solve the integral equation directly for real frequencies 
where the limit $\omega \rightarrow 0$ can be taken analytically. In practice, this approach is useful at high temperatures, where self-consistency no longer plays a role.

\section{Boltzmann-equation limit}

In the high-temperature limit $T \gg T_{F}$ the integral equations (38)-(43) can be solved by expanding in powers of the fugacity

$$
z=e^{\beta \mu}=\frac{4}{3 \sqrt{\pi}} \theta^{-3 / 2}+\mathcal{O}\left(\theta^{-3}\right) .
$$

To leading order in $z$, the pair propagator and on-shell self-energy are given by

$$
\begin{aligned}
& \Gamma^{\mathrm{ret}}(\boldsymbol{k}, \Omega)=-i \frac{4 \pi \hbar^{3} m^{-3 / 2}}{\sqrt{\hbar \Omega+2 \mu-\varepsilon_{\boldsymbol{k}} / 2}}+\mathcal{O}(z) \\
& \Sigma^{\mathrm{ret}}\left(\boldsymbol{p}, \epsilon=\varepsilon_{\boldsymbol{p}}-\mu\right)=i \frac{8 \varepsilon_{F}}{3 \pi} \frac{\operatorname{erf}\left(\sqrt{\pi} p / p_{T}\right)}{p / p_{F}}+\mathcal{O}(z) .
\end{aligned}
$$

In the case of on-shell fermions with $\boldsymbol{k}=\boldsymbol{p}_{1}+\boldsymbol{p}_{2}, \hbar \Omega+2 \mu=\varepsilon_{\boldsymbol{p}_{1}}+\varepsilon_{\boldsymbol{p}_{2}}$ the pair propagator reduces to the well-known scattering amplitude $f(q)=i / q$ at infinite scattering length of two particles in vacuum, with relative momentum $q$. Note that the exact leadingorder result for the on-shell fermionic self-energy contains a non-trivial error-function dependence on the ratio of the momentum $p$ to its thermal value $p_{T}$ that was missing in previous studies [60]. It is due to the square-root tail in the pair propagator and gives a noticeable correction at thermal momenta $p \simeq p_{T}$. Moreover, this form is indeed crucial to fulfill the condition of scale invariance, as will be discussed below.

The fermionic spectral function in the low fugacity, high temperature limit has most of the spectral weight concentrated in the coherent peak at $\epsilon=\varepsilon_{p}-\mu$. The peak width $\gamma_{p}=\operatorname{Im} \Sigma^{\mathrm{ret}}(\boldsymbol{p}, \epsilon)$ vanishes like $\varepsilon_{F} p_{F} / p \sim T^{-1 / 2}$ for typical momenta $p \approx p_{T}$, consistent with the assumption for the temperature dependence of the relaxation time introduced by Bruun and Smith [26]. This implies, in particular, that the fermionic quasiparticles become well-defined and thus a Boltzmann equation description is valid in the regime $\theta \gg 1$.

From a numerical, iterative solution of the integral equations (38)-(43) in the hightemperature limit we obtain $\eta /(\hbar n)=2.80(1)\left(T / T_{F}\right)^{3 / 2}$. This fixes the constant in the asymptotic behavior $\alpha(\theta)=$ const $\theta^{3 / 2}$ at large values of $\theta$ of the universal function introduced in (14). Within the error bars, the numerical value agrees with that obtained from a variational solution of the full Boltzmann equation, using higher Sonine polynomials [26, appendix]. The prediction of a simple power-law dependence of the shear viscosity $\eta(T) \sim T^{3 / 2}$ has recently been verified experimentally in a temperature range between $\theta \simeq 1.5$ and $\theta \simeq 7$ by measuring the expansion dynamics of a unitary gas released from an optical trap [61]. Very good agreement has been found also with the expected prefactor, thus considerably improving the situation compared to earlier measurements of the shear viscosity from the damping of the radial breathing mode [62].

Remarkably, the solution of the transport integral equation at high temperatures and small frequencies can also be obtained by a completely analytical approach. In fact, in the low fugacity limit, one can terminate the iterative procedure after the first 

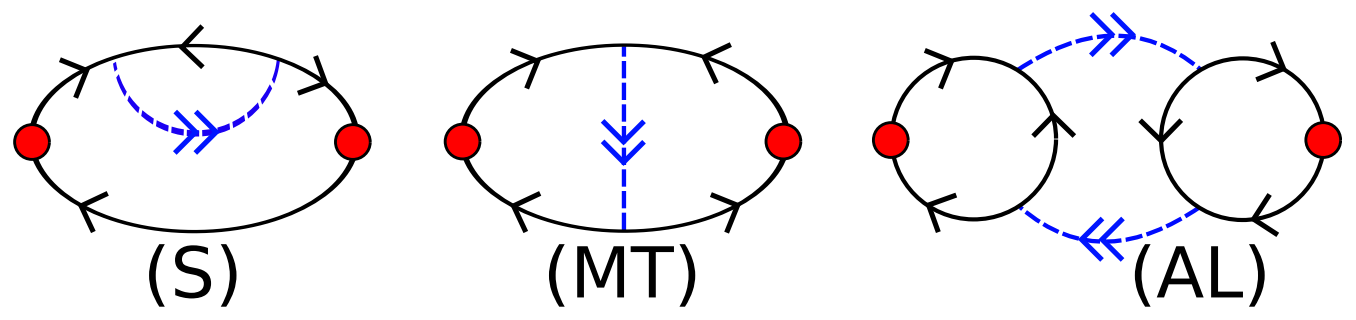

Figure 3: [color online] Diagrammatic contributions to the viscosity correlation function $\chi_{\ell}(\omega)$ at first order in the pair fluctuations: Self-energy (S), Maki-Thompson (MT) and Aslamazov-Larkin (AL) diagrams.

iteration step (correlation function to first order in the pair propagator) and resum via a memory function approach, a method that was developed in the context of electrical conductivities by Götze and Wölfle [63]. The first-order correlation function contains the diagrams for self-energy, Maki-Thompson and Aslamazov-Larkin contributions shown in Fig. 3. These diagrams are obtained by evaluating the transport equations (38)-(43) with the bare viscosity vertices $T_{\ell}^{(0)}, S_{\ell}^{(0)}$ on the right-hand side, and with bare fermionic propagators $G_{0}(p, \epsilon)$ with an additional impurity scattering rate $\gamma$, which is taken to zero after resummation.

Explicitly, to lowest order both in the fugacity and in the scattering rate, the bosonic vertex correction $S_{\ell}$ in equation (43) is given by

$$
S_{\ell=2}(k, \Omega+i 0, \Omega-i 0)=\frac{\varepsilon_{F}^{1 / 2} \varepsilon_{k} \sqrt{2(\hbar \Omega+2 \mu)-\varepsilon_{k}}}{32 \pi \gamma} .
$$

Here, $S_{\ell}$ in Fourier space (for notation see equation (B.5)) is continued to real frequency, with one retarded and one advanced boson line, $S_{\ell}(k, \omega+i 0, \Omega+\omega+i 0, \Omega-i 0) \rightarrow$ $S_{\ell}(k, \Omega+i 0, \Omega-i 0)$ in the limit of vanishing external frequency $\omega \rightarrow 0$, to obtain a function of wavenumber $k$ and real frequency $\Omega$. This bosonic viscosity vertex is used to compute the fermionic AL vertex correction in equation (41).

Next, the fermionic MT and AL viscosity vertices are continued to real frequencies, with one retarded and one advanced fermion line, $T_{\ell}(k, \omega+i 0, \epsilon+\omega+i 0, \epsilon-i 0) \rightarrow$ $T_{\ell}(k, \epsilon+i 0, \epsilon-i 0)$ in the limit of vanishing external frequency $\omega \rightarrow 0$. Finally, since the fermions are well-defined quasiparticles it is sufficient to evaluate the viscosity vertices on-shell $\left(\epsilon=\varepsilon_{\boldsymbol{p}}-\mu\right)$. Introducing a dimensionless momentum variable $y=p / \sqrt{2 m k_{B} T}=$ $\sqrt{\pi} p / p_{T}$, the resulting fermionic MT and AL vertex corrections are given by

$$
\begin{aligned}
& T_{\ell=2}^{\mathrm{MT}}(y)=\frac{z T^{2}}{\sqrt{\pi} \gamma}\left[-\frac{3}{4 y^{3}} \operatorname{erf}(y)+\frac{1}{\sqrt{\pi}}\left(1+\frac{3}{2 y^{2}}\right) e^{-y^{2}}\right] \\
& T_{\ell=2}^{\mathrm{AL}}(y)=\frac{z T^{2}}{\sqrt{\pi} \gamma}\left[\left(y+\frac{1}{y}+\frac{3}{4 y^{3}}\right) \operatorname{erf}(y)-\frac{3}{\sqrt{\pi}}\left(1+\frac{3}{2 y^{2}}\right) e^{-y^{2}}\right] .
\end{aligned}
$$

From the two fermionic vertex corrections and the self-energy contribution one obtains the leading correction to the shear viscosity $\eta(\omega \rightarrow 0)$ according to Fig. 3,

$$
\eta^{(1)}=\eta_{\mathrm{S}}^{(1)}+\eta_{\mathrm{MT}}^{(1)}+\eta_{\mathrm{AL}}^{(1)}=\frac{z^{2}(2 m)^{3 / 2} T^{7 / 2}}{15 \pi^{5 / 2} \hbar^{2} \gamma^{2}} \times \frac{-43-3+30}{8 \sqrt{2}}=-\frac{\sqrt{2} z^{2}(2 m)^{3 / 2} T^{7 / 2}}{15 \pi^{5 / 2} \hbar^{2} \gamma^{2}}
$$


which is negative because adding a scattering channel on top of the impurity scattering $\gamma$ lowers the viscosity. Now, the memory function approach effectively assumes that the first-order correction $\eta^{(1)}$ to the non-interacting viscosity

$$
\eta^{(0)}=\frac{z(2 m)^{3 / 2} T^{5 / 2}}{8 \pi^{3 / 2} \hbar^{2} \gamma}
$$

is the first term of a geometric series,

$$
\begin{aligned}
& \eta=\eta^{(0)}+\eta^{(1)}+\cdots \approx \eta^{(0)}\left[1-\eta^{(1)} / \eta^{(0)}\right]^{-1} \\
& \stackrel{\gamma \rightarrow 0}{\longrightarrow}-\frac{\left(\eta^{(0)}\right)^{2}}{\eta^{(1)}}=\frac{15 \hbar k_{F}^{3}}{64 \sqrt{2 \pi}} \theta^{3 / 2} .
\end{aligned}
$$

Normalized to the particle density, the resulting shear viscosity at high temperatures is thus given by

$$
\eta=\frac{45 \pi^{3 / 2}}{64 \sqrt{2}} \hbar n \theta^{3 / 2} \approx 2.77 \hbar n \theta^{3 / 2} .
$$

This analytical result is in perfect agreement with that obtained from the numerical solution of the full transport equations above and agrees also with the result obtained from the Boltzmann equation, using the standard variation of the distribution function $\delta f \propto v_{x} p_{y}[64,65]$. Our evaluation of the Kubo formula within the T-matrix approximation thus recovers the exact Boltzmann equation result at high temperatures and low frequencies. It is important to note that a calculation of the shear viscosity which uses a nontrivial Ansatz for the fermionic spectral functions but does not include vertex corrections [26], gives the correct $T^{3 / 2}$ asymptotic power law at unitarity, however the associated prefactor 1.06 is far too small. Indeed, the single-particle scattering time underestimates the transport scattering time $\tau_{\eta}$ by a factor of almost 2.6. The $\mathrm{V}$ and AL vertex corrections in the Kubo formula are therefore crucial even in the classical limit. They are equivalent to solving the full Boltzmann equation, not only its relaxation-time approximation.

Moreover, neglecting vertex corrections violates the Ward identities associated with scale invariance and thus leads to a finite bulk viscosity at unitarity. Indeed, it is straightforward to show that only the sum of the three first-order corrections to the bulk viscosity (order $\gamma^{-2}$ in the impurity scattering rate)

$$
\zeta^{(1)}=\zeta_{\mathrm{S}}^{(1)}+\zeta_{\mathrm{MT}}^{(1)}+\zeta_{\mathrm{AL}}^{(1)} \equiv 0
$$

vanishes identically, as expected for a scale-invariant system. The error-function term in the fermionic self-energy (50) is crucial for this cancellation, as well as for satisfying the Ward identities derived in Appendix B.

\section{Results for the shear viscosity}

As the temperature is lowered towards the degeneracy temperature $T_{F}$, the fugacity ceases to be a good expansion parameter, reaching $z=1$ at around $T \approx 0.6 T_{F}$. In a 
perturbative approach, this regime can be approached from the ideal gas via the virial expansion $[66,67,68]$. The known exact values of the second and third virial coefficient can then be used for a calibration of measurements of the equation of state of the unitary gas $[69,70,71]$. For a calculation of dynamical properties like the viscosity in the regime $0.15<\theta \lesssim 1$, where the gas is degenerate but still in a non-superfluid state, it is necessary to use a non-perturbative approach that also provides information about dynamical correlation functions. As discussed in section 5, this can be achieved by a self-consistent Luttinger-Ward formulation of the many-body problem, which allows to determine not only thermodynamic properties of the unitary gas at arbitrary temperatures [58] but also the fermionic spectral functions [59]. In the relevant regime $0.15<\theta \lesssim 1$ just above the superfluid transition temperature, they exhibit a substantial broadening, which is of order of the Fermi energy itself [59]. As a result, the fermionic quasiparticles are no longer well-defined. The integral equations (38)-(43) for the viscosity vertices as functions of (radial) distance and imaginary time can then only be solved numerically. In practice, the self-consistent set of integral equations is iterated until convergence is reached (typically after a few steps); then the correlation function $\chi_{x y, x y}\left(i \omega_{m}\right)=\chi_{\ell=2}\left(i \omega_{m}\right) / 15$ is continued analytically to real frequencies.

As a first step to evaluate the shear viscosity for real frequencies, we perform the analytic continuation by the Padé method, using the first 300 Matsubara frequencies [59]. The resulting $\eta(\omega)$ curves are shown in Fig. 4. They exhibit a clear Drude peak, which crosses over into a $\omega^{-1 / 2}$ tail at large $\omega$. Unfortunately, it is difficult to extract a precise value for the transport scattering time at large temperatures by this method. To obtain reliable results for the frequency-dependent viscosity, we therefore use a simple model function of the form

$$
\chi_{x y, x y}\left(i \omega_{m}\right)=\frac{W-\sqrt{2} \hbar^{3 / 2} C_{\eta} m^{-1 / 2} \tau_{\eta}\left|\omega_{m}\right|^{3 / 2}}{1+\tau_{\eta}\left|\omega_{m}\right|}+\frac{4 \hbar^{2} C \Lambda}{15 \pi^{2} m} .
$$

It describes the dependence on the Matsubara frequencies found numerically from the self-consistent solution of the transport equation very well for arbitrary temperatures above $T_{c}$. The additive contribution in equation (56) which is linear in the momentum cutoff $\sim C \Lambda$ has been derived in (34). It is, however, irrelevant for the associated viscosity spectral function

$$
\begin{aligned}
\eta(\omega)=\frac{\operatorname{Im} \chi_{x y, x y}^{\mathrm{ret}}(\omega)}{\omega}=\frac{W \tau_{\eta}}{1+\left(\omega \tau_{\eta}\right)^{2}}+ & \frac{\hbar^{3 / 2} C_{\eta}}{\sqrt{m \omega} \frac{\omega \tau_{\eta}\left(1+\omega \tau_{\eta}\right)}{1+\left(\omega \tau_{\eta}\right)^{2}}} \\
& \longrightarrow \begin{cases}W \tau_{\eta}+\hbar^{3 / 2} C_{\eta} \tau_{\eta} \sqrt{\omega / m} & \text { as } \omega \rightarrow 0 \\
\hbar^{3 / 2} C_{\eta} / \sqrt{m \omega} & \text { as } \omega \rightarrow \infty\end{cases}
\end{aligned}
$$

which exhibits a Drude-like transport peak and a $1 / \sqrt{\omega}$ tail, as expected on general grounds. This viscosity function is in very good agreement with the Padé results shown in Fig. 4 and has the advantage that the resulting $\tau_{\eta}$ from the fitting function agrees perfectly with the exact result in the classical limit. Within this Ansatz, the frequencydependent shear viscosity is characterized by three parameters: the total Drude weight $W(T)$, the viscous transport scattering time $\tau_{\eta}(T)$ and a tail coefficient $C_{\eta}(T)$. In practice, since both the Drude weight and the tail coefficient are fixed by the equilibrium variables $p$ and $C$ via the exact sum rule (13), the transport scattering time remains as 


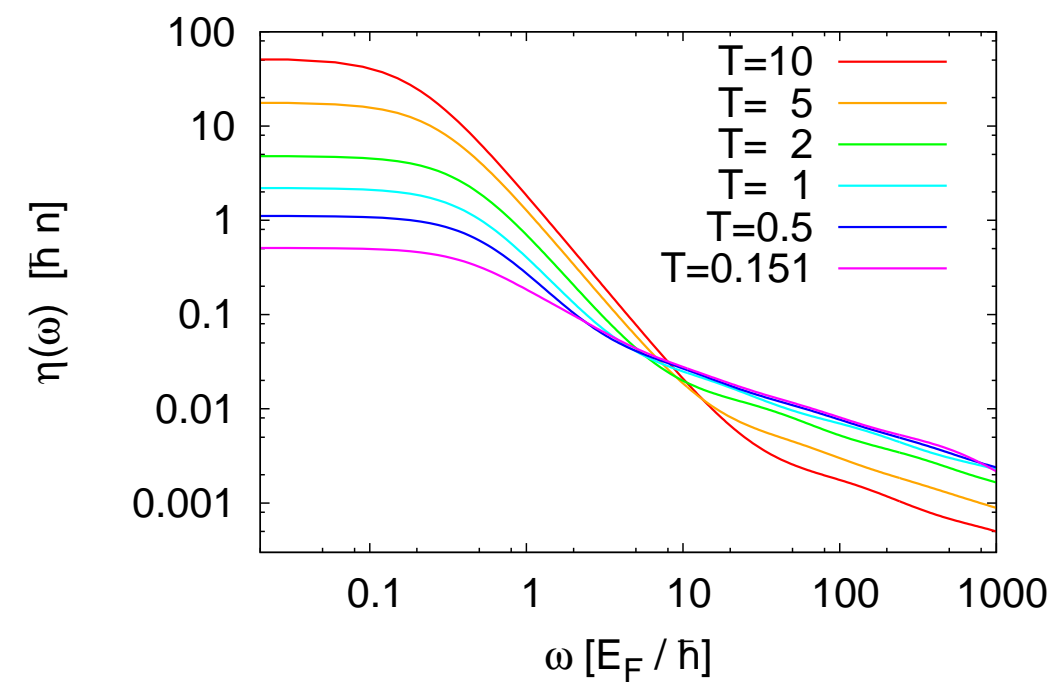

Figure 4: [color online] Shear viscosity spectral function $\eta(\omega)$ for different temperatures (on the left side of the plot, temperature decreases from top to bottom; on the right side the order is opposite). The analytical continuation is performed by the Padé method.

the single adjustable parameter. Note that the dc-viscosity in equation (57) has precisely the form that is assumed in Maxwell's theory of strongly viscous fluids, with $W \equiv G_{\infty}$ playing the role of the high-frequency shear modulus [40]. An unexpected feature of our model function (57) is the presence of a $\sqrt{\omega}$ singularity at low frequencies, whose weight is too small, however, to be seen in Fig. 4 . As a result, there is a negative $t^{-3 / 2}$ long-time tail in the relaxation of shear stress, similar to that arising in both classical and quantum liquids due to mode coupling effects $[40,72]$. If we insert the model function into the left-hand side of the sum rule (13),

$$
\frac{2}{\pi} \int_{0}^{\infty} d \omega\left[\frac{W \tau_{\eta}}{1+\left(\omega \tau_{\eta}\right)^{2}}+\frac{\hbar^{3 / 2} C_{\eta}}{\sqrt{m \omega}} \frac{\omega \tau_{\eta}\left(1+\omega \tau_{\eta}\right)}{1+\left(\omega \tau_{\eta}\right)^{2}}-\frac{\hbar^{3 / 2} C}{15 \pi \sqrt{m \omega}}\right]=W
$$

the second term of the model function and the subtraction of the high-frequency tail integrate to zero exactly if $C_{\eta}=C /(15 \pi)$, for any value of $\tau_{\eta}$. The frequency integral thus yields the Drude weight $W$, which is equal to the equilibrium pressure $p$ at unitarity by the exact sum rule (13).

As shown in Fig. 5, the total weight of the Drude peak $W$ agrees remarkably well with the equilibrium pressure of the unitary gas for all temperatures, from the classical limit down to the superfluid transition at $T_{c} \simeq 0.15 T_{F}$. Here and in the following, all thermodynamic properties of the unitary Fermi gas are taken from the equivalent Luttinger-Ward calculation of our previous work in Ref. [58]. The viscous transport scattering time $\tau_{\eta}$ versus temperature is shown in Fig. 6: for large temperatures it approaches $\tau_{\eta} \sim \theta^{1 / 2} \hbar / \varepsilon_{F}$, consistent with equation (54) and the result obtained from kinetic theory [26]. For temperatures near $T_{c}$, it is enhanced by a factor of two compared with a simple extrapolation of the high temperature result. Qualitatively, there are two 


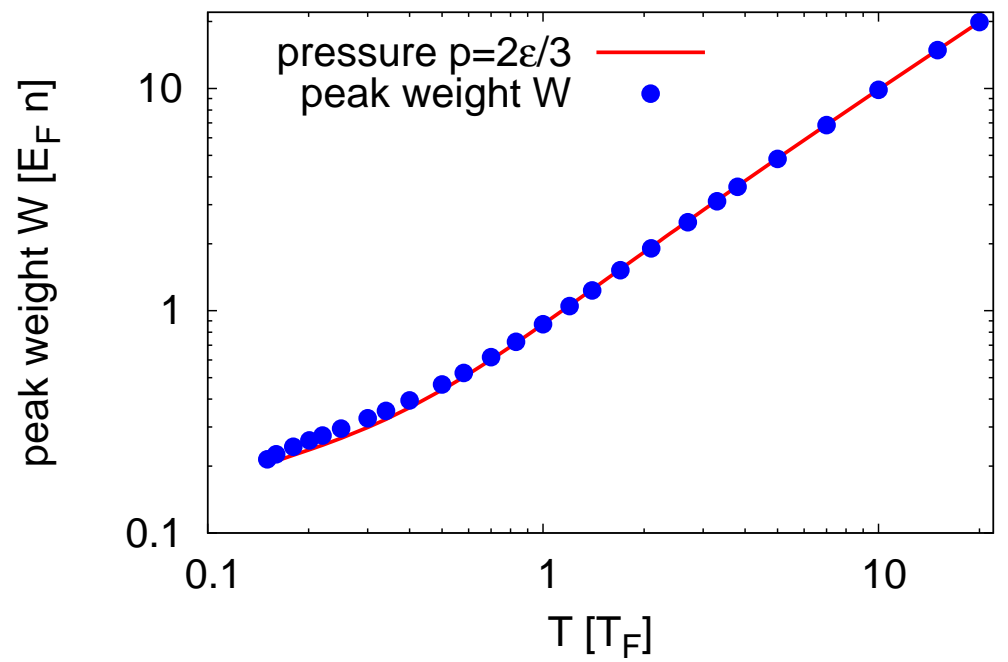

Figure 5: [color online] Weight $W$ of the Drude peak in the shear viscosity spectral function $\eta(\omega) \approx$ $W \tau_{\eta} /\left[1+\left(\omega \tau_{\eta}\right)^{2}\right]$ as a function of temperature. The pressure $p(T)$ (red line) is from Ref. [58].

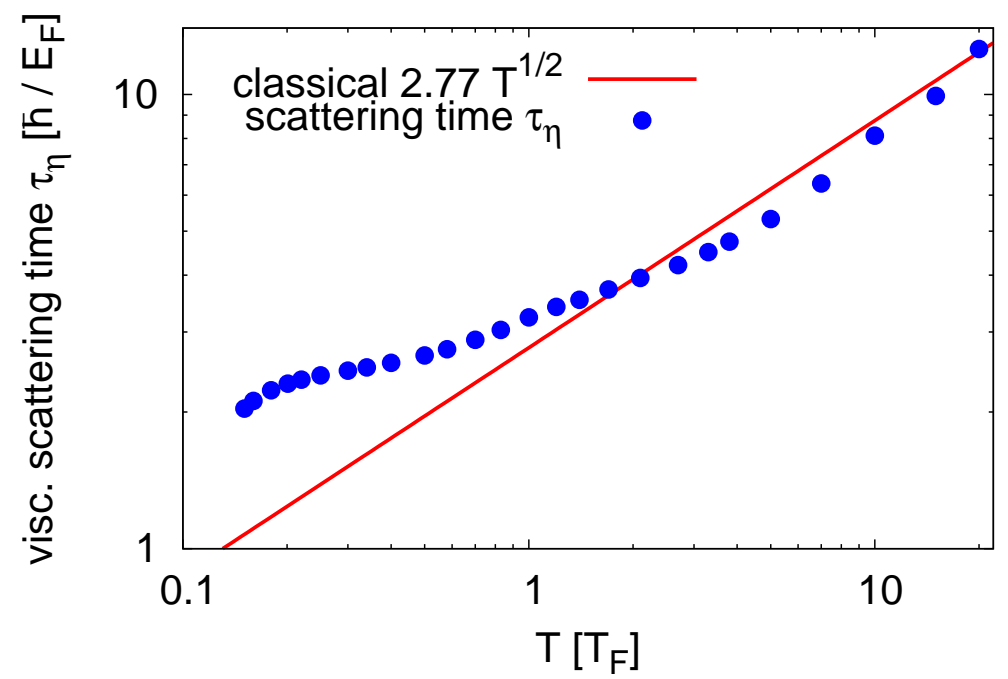

Figure 6: [color online] Viscous scattering time $\tau_{\eta}$ of the Drude peak in the shear viscosity spectral function $\eta(\omega) \approx W \tau_{\eta} /\left[1+\left(\omega \tau_{\eta}\right)^{2}\right]$ as a function of temperature. The classical limit (red line) is from equation (54). 


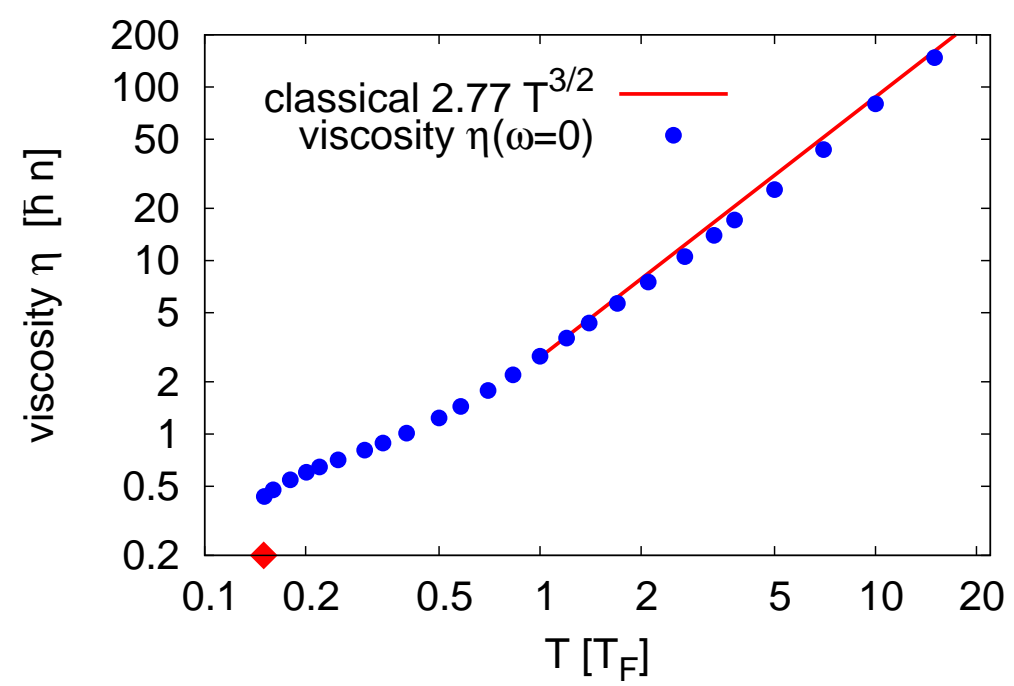

Figure 7: [color online] Static shear viscosity $\eta(\omega=0)$ as a function of temperature. The classical limit $\eta \simeq 2.77\left(T / T_{F}\right)^{3 / 2}$ (red line) is from equation (54). The red diamond indicates $T_{c} \simeq 0.15 T_{F}$.

competing effects on the scattering time as the temperature is lowered from the classical limit: Pauli blocking reduces the phase space and increases $\tau_{\eta}$, while pairing fluctuations enhance scattering and lower $\tau_{\eta}$. In a kinetic theory approximation, both effects nearly balance and $\tau_{\eta}$ remains essentially at the classical value down to $T_{c}$ [73]. By contrast, as is evident from Fig. 6, our evaluation of the Kubo formula within a self-consistent T-matrix approximation predicts strong deviations from kinetic theory in the relevant regime $\theta \lesssim 1$. Kinetic theory is clearly inapplicable for a degenerate gas and indeed the width $\hbar / \tau_{\eta} \approx 0.5 k_{B} T_{F}$ is more than a factor three larger than the thermal energy at the lowest temperature $T=T_{c}$ that is studied here.

The static value $\eta=\eta(\omega=0)$ of the shear viscosity is shown in Fig. 7. It exhibits a monotonic dependence on temperature, reaching $\eta \sim 0.5 \hbar n$ at the superfluid transition temperature, still with a positive slope. As discussed in section 4, one expects a diverging positive derivative of $\eta(T)$ just below $T_{c}$. As a result, the minimum of the viscosity is expected below the superfluid transition, a regime that is not accessible within our present approach. The strong rise of the shear viscosity predicted for $T \ll T_{c}$ in equation (15), however indicates that the minimum value of the shear viscosity for the unitary gas is close to the value reached at $T_{c}$. The dimensionless function $\alpha(\theta)$ thus has a minimum value of order $\alpha_{\eta}^{\min } \simeq 0.5$, a value that is smaller than any other that is found for non-relativistic fluids [3]. Recalling the connection (12) between shear viscosity and the associated diffusion constant $D_{\eta}$, which is identical with the kinematic shear viscosity $\nu$ for a normal fluid, the minimum value for $\eta$ is equivalent to a minimum value $D_{\eta} \simeq 0.5 \hbar / \mathrm{m}$ of the shear diffusion constant, which only involves Planck's constant and the particle mass. Bounds of a similar form are also expected for other diffusion constants in the unitary gas, for instance heat or particle diffusion. In fact, the latter has been measured recently by studying the equilibration dynamics after the two spin components are separated in 


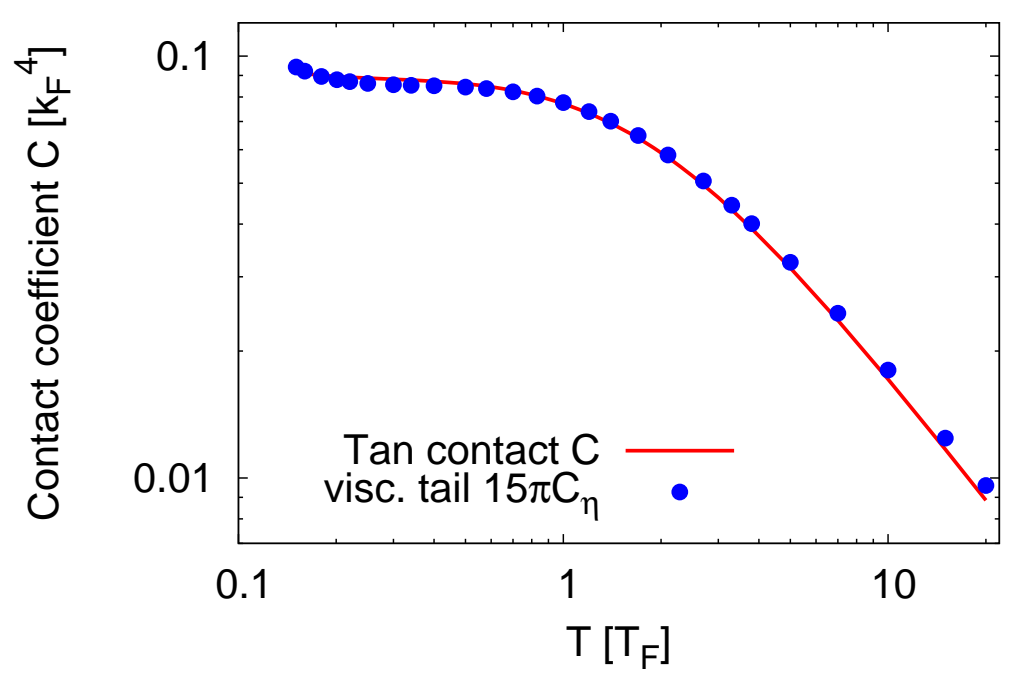

Figure 8: [color online] Tail of the shear viscosity spectral function $\eta(\omega) \approx \hbar^{3 / 2} C_{\eta} / \sqrt{m \omega}$ as a function of temperature. The contact $C$ (red line) is computed numerically according to Ref. [59] and follows the high-temperature asymptotics in equation (60).

a trap [74]. In the degenerate regime, the observed diffusion constant is again of order $\hbar / m$. Concerning heat transport, which so far has neither been calculated nor measured for the unitary gas, it is interesting to note that the associated diffusion constant $D_{T}$ is predicted to be identical with the shear diffusion constant $D_{\eta}$ for non-relativistic fluids that exhibit a gravity dual [75]. A comparison of thermal diffusion and shear viscosity would thus provide a measure of how close the unitary gas is to such an idealized system, whose Prandtl number $\operatorname{Pr}=D_{\eta} / D_{T}$ is identically equal to one at all temperatures. In the non-degenerate regime $\theta \gg 1$ one expects in fact that $\operatorname{Pr} \approx 2 / 3$, a result that is essentially universal for dilute classical gases [39].

The tail in the viscosity spectral function (57) has the general form

$$
\eta(\omega)=\frac{\hbar^{3 / 2} C_{\eta}}{\sqrt{m \omega}}+\mathcal{O}\left(\omega^{-3 / 2}\right) \quad\left(\omega \gg \omega_{x}\right)
$$

above a crossover scale $\omega_{x}$ which we define as the intersection point of the Drude and square-root asymptotics. The crossover frequency $\omega_{x}(T)$ at large temperatures scales like $\omega_{x} \sim T$ which agrees with the extension of the Drude peak expected from kinetic theory, $\omega \leq T[3]$. Note that near $T_{c}$ the peak viscosity is still about five times higher than the viscosity $\eta\left(\omega_{x}\right)$ at the onset of the tail, but the transport peak is nevertheless much less pronounced as compared to higher temperatures. In Fig. 8 we plot the tail coefficient $C_{\eta}$ extracted from our numerical results for the frequency dependent shear viscosity as a function of temperature. Apparently, the connection

$$
C_{\eta}=\frac{C}{15 \pi}
$$


between the tail coefficient $C_{\eta}(T)$ and the Tan contact density $C(T)$ that follows from the exact sum rule (13) is fulfilled very accurately at all temperatures (for an alternative derivation of the relation (59) see Appendix C). To understand the temperature dependence of the tail coefficient, which saturates for low temperatures and decreases as $\sim 1 / T$ for large temperatures $T \gtrsim T_{F}$, we note that the contact density is defined via the asymptotics of the momentum distribution, $n_{\sigma}(k) \rightarrow C / k^{4}$ for large wavenumbers $k \gg k_{F}$. Alternatively, the contact density can be determined from the vertex function (C.4) as $\hbar^{4} C=-m^{2} \Gamma_{X^{\prime}=X^{+}}$[58]. At high temperatures $T \gg T_{F}$ this can be evaluated analytically and leads to

$$
C(T)=\frac{4 m^{2} z^{2} T^{2}}{\pi \hbar^{4}}=\frac{8 \pi^{2} \hbar^{2} n^{2}}{m T}=\frac{16 k_{F}^{4}}{9 \pi^{2} \theta},
$$

which agrees precisely with the result obtained in Ref. [76] (note the different definition of the contact in this work which accounts for an apparent difference by a factor of $4 \pi^{2}$ ). This asymptotic behavior is in perfect agreement with our numerical results in Fig. 8. An alternative way to infer the high frequency behavior of the shear viscosity is based on the relation $[28]$

$$
\eta(\omega)=\lim _{q \rightarrow 0} \frac{3 \omega^{3}}{4 \hbar q^{4}} \operatorname{Im} \chi_{\rho \rho}(\boldsymbol{q}, \omega)
$$

between the frequency-dependent shear viscosity and the mass-density correlation function $\chi_{\rho \rho}(\boldsymbol{q}, \omega)$, a relation that is valid at all frequencies. As shown by Son and Thompson [77], the density correlation function at large frequencies

$$
\operatorname{Im} \chi_{\rho \rho}(\boldsymbol{q}, \omega \rightarrow \infty)=\frac{4 \hbar^{5 / 2} q^{4} C}{45 \pi m^{1 / 2} \omega^{7 / 2}}
$$

is again fully determined by the Tan contact $C$. The resulting coefficient $C_{\eta}$ in the highfrequency tail of the shear viscosity agrees precisely with that in equation (59) above.

Our result for the temperature-dependent shear viscosity can now be combined with the known value of the entropy density [58] to determine the ratio $\eta / s$ in the normal fluid regime of the unitary gas. As shown in Fig. 9, this ratio exhibits a very shallow minimum around $T \approx 0.3-0.4 T_{F}$, below which $\eta / s$ increases very slowly. The precise location of the minimum clearly depends sensitively on how accurate the results for both the viscosity and entropy are in this regime. On quite general grounds, it is likely that the minimum in $\eta / s$ is close to the superfluid transition temperature $T_{c} \simeq 0.15 T_{F}$, and that $\eta / s$ is monotonically increasing as the temperature is lowered in the superfluid regime, eventually crossing over to the steep increase of equation (16) as the temperature approaches zero. The fact that our diagrammatic calculation gives results, e.g., for the critical temperature and the associated entropy density $s \simeq 0.7 n k_{B}[58]$ which agree well with precise numerical results [78], suggests that our ratio $\eta / s$ provides a quantitatively reliable estimate, despite the fact that the precise location of the minimum is difficult to determine. Granting that our value $\eta / s \simeq 0.6 \hbar / k_{B}$ for the minimum is close to the exact result, we conclude that the ratio $\eta / s$ for the unitary Fermi gas remains a factor of about seven above the KSS bound, somewhat larger than the experimental estimates for the quark-gluon plasma [3]. 


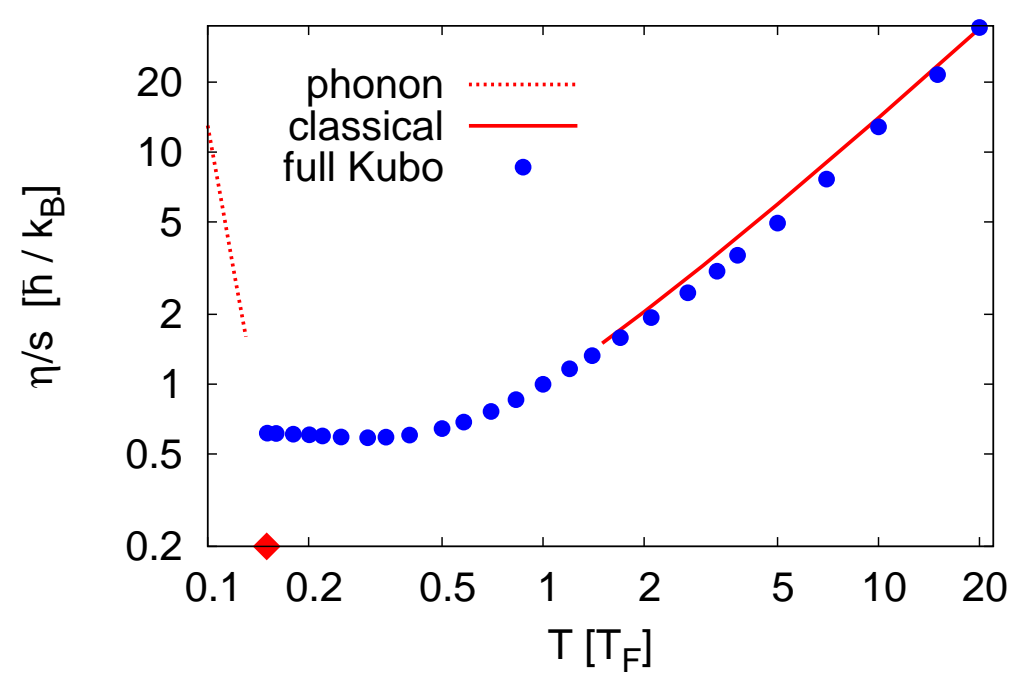

Figure 9: [color online] Shear viscosity to entropy ratio $\eta / s$ (blue circles) in comparison with known asymptotes. The dashed red line on the left is the phonon contribution $\eta / s \sim\left(T / T_{F}\right)^{-8}$ in equation (16), the solid red line on the right the classical limit (54) divided by the classical entropy from the Sackur-Tetrode formula. The red diamond indicates $T_{c} \simeq 0.15 T_{F}$.

\section{Conclusions}

Using a diagrammatic approach that follows the classic Baym-Kadanoff conserving approximation to calculate transport properties of fermionic quantum liquids, we have determined the frequency dependent shear viscosity $\eta(\omega)$ of the unitary Fermi gas in its normal phase $\theta>\theta_{c} \approx 0.15$. With decreasing temperatures, the Drude peak in the viscosity is smeared out and spectral weight is transferred to the $1 / \sqrt{\omega}$ tail, precisely following the increase in the Tan contact. In the degenerate gas regime $\theta \lesssim 1$, transport of transverse momentum cannot be described in terms of a quasiparticle picture because the viscosity spectral function is much broader than the thermal energy. Our results for $\eta(\omega)$ are in perfect agreement with the exact sum rule (13) which, apart from numerical factors, agrees with the one derived by Taylor and Randeria [28]. In particular, the weight of the Drude peak, which is effectively the fluid's high-frequency shear modulus, is identically equal to the gas pressure at all temperatures above $T_{c}$, while the coefficient $C_{\eta}=C /(15 \pi)$ of the high-frequency $1 / \sqrt{\omega}$ tail agrees with the Tan contact density.

Our dynamic shear viscosity exhibits a $\sqrt{\omega}$ singularity both at high and low frequencies. While the high-frequency tail is a consequence of the zero-range interaction, providing a direct measure of the contact, the physical interpretation and a detailed theory of the singularity at low frequencies that is related to the well-known $t^{-3 / 2}$ long-time tails due to mode coupling effects remains an open problem. Note that within our formulation, the sign of the $\sqrt{\omega}$ singularity is positive, as is found for quantum liquids [72], while the standard long-time tails in classical liquids lead to a negative contribution.

The proper treatment of symmetries and the inclusion of vertex corrections guarantees that our diagrammatic approach leads to a bulk viscosity that vanishes identically. In 
particular, our approximation exactly satisfies the Tan energy relation (28) and the adiabatic sweep theorem [44]. Moreover, it reproduces the high-temperature Boltzmann equation result for the shear viscosity by Bruun and Smith [26]. The accuracy of our approximation in the most interesting regime just above the superfluid transition is difficult to estimate a priori. The agreement with the non-perturbative viscosity sum rule, however, suggests that our results for the viscosity are quantitatively reliable down to the superfluid transition. The dc-viscosity is found to decrease monotonically with temperature, reaching a value $\eta \simeq 0.5 \hbar n$ near $T_{c}$. Equivalently, the unitary gas near $T_{c}$ exhibits a quantum limited shear diffusion constant (or kinematic viscosity) $D_{\eta} \simeq$ $0.5 \hbar / m$. The ratio $\eta / s$ between shear viscosity and entropy density exhibits a minimum slightly above the superfluid transition temperature. The corresponding value $\eta / s \simeq$ $0.6 \hbar / k_{B}$ is a factor of about seven above the KSS bound, the smallest value found for non-relativistic fluids so far.

There are, of course, a number of problems that remain open. For the unitary gas, precise results for the shear viscosity near $T_{c}$ and also immediately below the superfluid transition are required to obtain definite predictions for the location and the associated value of the minimum in both $\eta$ and in $\eta / s$. Similarly, a quantitative understanding of current experiments on the inter-diffusion of the two spin components [74] requires to study particle transport by a calculation of the conductivity from the longitudinal current correlation function. Another transport coefficient that may be determined by an extension of our present method is the heat diffusion constant. Its ratio with the shear diffusion constant determines the Prandtl number, which is equal to unity in nonrelativistic, conformal field theories with a holographic dual [75].

Finally, it is of interest to study the situation away from unitarity, where scale invariance is broken and a finite bulk viscosity appears. Since both $\eta$ and the ratio $\eta / s$ will exhibit a minimum as a function of temperature for arbitrary values of $1 / k_{F} a$ along the BCS-BEC crossover, this would allow to answer the question whether the minimum found at unitarity indeed gives the lowest possible value and thus, whether scale invariance is an important ingredient for a minimum in $\eta / s$ in a non-relativistic context. In fact, the entropy density at $T_{c}$ has its largest value not at unitarity but near $1 / k_{F} a \approx 1$ [58]. A calculation of the viscosity along the complete BCS-BEC crossover has been performed in one dimension, where a finite bulk viscosity exists at $T=0$ [79]. In this case, the zero temperature viscosity is actually smallest in the BEC limit.

We are grateful for a number of fruitful discussions with Dietrich Belitz, Jens Braun, Wolfgang Götze, John McGreevy, Yusuke Nishida, Mohit Randeria, Achim Rosch, Manfred Salmhofer, Jörg Schmalian, Richard Schmidt, Thomas Schäfer and Martin Zwierlein. This work has been supported in part by the DFG research unit "Strong Correlations in Multiflavor Ultracold Quantum Gases".

\section{Appendix A. Viscosity of a Lennard-Jones fluid}

In this Appendix we give an elementary argument, based on dimensional analysis only, for the quite surprising observation that the KSS bound which arises from genuine quantum field theory considerations, even applies to classical fluids, whose viscosity minimum apparently cannot depend on $\hbar$. An example is water, a rather complex fluid, that has a minimum viscosity near its critical point at $T=650 \mathrm{~K}$. Since a viscosity has 
units energy density times seconds, the ratio $\alpha_{\eta}=\eta /(\hbar n)$ is a pure number, which happens to be of order one, $\alpha_{\eta} \simeq 32$ at the viscosity minimum. This appears to indicate that quantum mechanics determines the minimum viscosity of water despite the fact that the thermal wavelength $\lambda_{T}$ near its critical point is much smaller than the average interparticle spacing $n^{-1 / 3}$.

To understand the origin of this surprising fact, consider a simple model for a classical fluid, which assumes pairwise central forces that are derived from a Lennard-Jones potential

$$
\Phi(r)=4 \varepsilon\left[\left(\frac{\sigma}{r}\right)^{12}-\left(\frac{\sigma}{r}\right)^{6}\right] .
$$

This potential is, of course, not a realistic model for such complex fluids as water, yet it provides a rather good quantitative description of simple fluids like Argon [40]. The two parameters $\sigma$ and $\varepsilon$ of this potential provide the characteristic scales for length and energy. As a result, the equation of state $p(n, T)=\varepsilon / \sigma^{3} p^{\star}\left(n \sigma^{3}, T / \varepsilon\right)$ is completely determined by a universal function $p^{\star}\left(n^{\star}, T^{\star}\right)$ of the dimensionless density $n^{\star}=n \sigma^{3}$ and the dimensionless temperature $T^{\star}=T / \varepsilon$. This provides an explanation for the law of corresponding states for different fluids that are reasonably well described by an interaction of the Lennard-Jones type. To evaluate dynamic correlation functions like the viscosity from a (classical) Kubo formula for this Lennard-Jones fluid requires in addition the particle mass $m$, which fixes a characteristic time scale $\tau=\left(m \sigma^{2} / \varepsilon\right)^{1 / 2}$. By purely dimensional arguments the shear viscosity of the Lennard-Jones fluid is then of the form

$$
\eta_{\mathrm{LJ}}(n, T)=\frac{\varepsilon \tau}{\sigma^{3}} \eta^{\star}\left(n^{\star}, T^{\star}\right)
$$

with a universal function $\eta^{\star}\left(n^{\star}, T^{\star}\right)$. In fact, equation (A.2) also applies for the bulk viscosity $\zeta$, which is typically of the same order as $\eta$ in classical fluids [40]. In the high-temperature, gaseous limit $T^{\star} \gg 1$ this function can be calculated from kinetic theory, where $\eta(T) \simeq\langle p\rangle / \sigma(T)$ is a ratio between an average momentum and a thermally averaged cross section $\sigma(T)$. For large temperatures, the cross section $\sigma(T) \sim p_{T}^{-4 / n}$ scales with an inverse power of the thermal momentum $p_{T}$. The exponent is determined by the short range repulsive potential $\Phi(r \rightarrow 0) \sim r^{-n}$. For a Lennard-Jones fluid with $n=12$, the universal function $\eta^{\star}\left(n^{\star}, T^{\star}\right) \sim\left(T^{\star}\right)^{2 / 3}$, therefore, exhibits a power-law increase with temperature, independent of density. At low temperatures the viscosity will again increase and eventually the response $\eta(\omega) \sim i / \omega$ becomes purely reactive at the liquid-to-solid transition, which appears at densities $n^{\star} \gtrsim 0.86[40]$ (Note that this is the density at the freezing point, which is smaller than the melting point density due to the first order nature of the transition. Note also that a gas of hard spheres with diameter $\sigma$ reaches close packing only at $n^{\star}=\sqrt{2}$ ). Numerical results for the dimensionless function $\eta^{\star}$ are available from molecular-dynamics simulations. For the Lennard-Jones fluid, they were first performed by Levesque et al. [80]. For example, the resulting value is $\eta^{\star}\left(n^{\star}=0.72, T^{\star}=0.84\right)=3.29$ near the triple point and close to the solidification line [81]. The minimum of the viscosity is necessarily reached near the gas-liquid transition, which lies between the triple and the critical point. For the Lennard-Jones fluid, the dimensionless densities and temperatures at these points are of order unity: $n_{t}^{\star} \simeq 0.85, T_{t}^{\star} \simeq 0.68$ and $n_{c}^{\star} \simeq 0.36, T_{c}^{\star} \simeq 1.36$, respectively [40]. From equation (A.2), it is thus obvious that the minimum value of the shear viscosity of a 
Lennard-Jones liquid

$$
\eta_{\mathrm{LJ}}^{\min }=\operatorname{const} \frac{\sqrt{m \varepsilon}}{\sigma^{2}}
$$

is fixed by the microscopic ratio $\sqrt{m \varepsilon} / \sigma^{2}$ with a universal prefactor of order unity, that unfortunately seems not to have been calculated so far. Clearly, this minimum value does not contain $\hbar$. Indeed, the condition for the applicability of classical physics to describe the behavior near the gas-liquid transition of the Lennard-Jones fluid is $\lambda_{T} \ll n^{-1 / 3} \simeq \sigma$, which implies that $\sqrt{m \varepsilon} \gg \hbar / \sigma$. The minimum value of the viscosity is therefore much larger than $\hbar / \sigma^{3} \simeq \hbar n$ as long as the standard de Boer parameter

$$
\Lambda^{\star}=\frac{\hbar}{\sigma \sqrt{m \varepsilon}}
$$

is very small. In practice, however, the de Boer parameter is still appreciable unless one considers fluids of very heavy elements like Xe. The associated conversion factor from equation (A.3) to a purely quantum limited viscosity $\eta=\alpha_{\eta} \hbar n$ is therefore not that large. Stated differently, for most fluids the zero-point energy $\hbar^{2} / m \sigma^{2}$ associated with the typical length scale of interatomic potentials is not much smaller than the well depth $\varepsilon$. This is a reflection of the fact that, after all, it is the combination of the quantum mechanical van der Waals interaction at large separations and the short range scale $\sigma$ that arises from the Pauli principle which determines the ratio $\sqrt{m \varepsilon} / \sigma^{2}$. It is, therefore, quantum mechanics which eventually fixes the minimum value of the viscosity of classical liquids to be of order $\hbar$ times the liquid density. With a corresponding entropy density $s \simeq k_{B} n$, this immediately yields a minimum value of $\eta / s$ that is not too far above the KSS bound, despite the fact that both the viscosity and the entropy may be inferred by completely classical considerations.

\section{Appendix B. Scale invariance and Ward identities}

In this Appendix we show that scale invariance and the associated continuity equation for the conserved dilatation current imply Ward identities which relate the fermionic and bosonic bulk viscosity vertices to the respective single-particle Green's functions. As a consequence the bulk viscosity vanishes identically in our approach as required by symmetry. In particular this allows us to check the consistency of the viscosity vertices that have been computed explicitly.

The trace of the stress tensor defined in equations (7) and (17),

$$
\Pi_{i i}(\boldsymbol{q}=0, t)=\Pi_{\ell=0}(t)=\sum_{\boldsymbol{p} \sigma} 2 \varepsilon_{p} c_{\boldsymbol{p} \sigma}^{\dagger} c_{\boldsymbol{p} \sigma}+\int d^{3} x \int d^{3} r 2 V(\boldsymbol{r}): n_{\uparrow}(\boldsymbol{x}) n_{\downarrow}(\boldsymbol{x}+\boldsymbol{r}):=2 H
$$

is twice the Hamiltonian in scale-invariant models $[82,21]$. The expectation value of this operator equation yields immediately $3 p=2 \epsilon[23]$, as shown explicitly in equation (28). The fermionic bulk viscosity response function $\tilde{T}_{\ell=0}$ in equation (31) can thus be written in imaginary time $\tau$ as

$$
\tilde{T}_{\ell=0}\left(\tau X_{1} X_{1}^{\prime}\right)=2\left\langle\mathcal{T} H(\tau) \psi\left(X_{1}\right) \psi^{\dagger}\left(X_{1}^{\prime}\right)\right\rangle .
$$


The effects of scale invariance are already incorporated in equation (B.1), and the evaluation of expectation values of the Hamiltonian yields a time derivative [54, equation $(28)]$

$$
\frac{d}{d \tau}\langle H(\tau)\rangle_{U}=\int d^{3} r \sum_{X^{\prime}}\left[U_{X X^{\prime}} \frac{\partial}{\partial \tau} G_{X^{\prime} X}+U_{X^{\prime} X} \frac{\partial}{\partial \tau} G_{X X^{\prime}}\right]
$$

where $X=(\boldsymbol{r}, \tau), X^{\prime}=\left(\boldsymbol{r}^{\prime}, \tau^{\prime}\right)$ and the external bilocal field $U_{X X^{\prime}}$ couples to fermion bilinears via a term $\sum_{X X^{\prime}} \psi^{\dagger}(X) U_{X X^{\prime}} \psi\left(X^{\prime}\right)$ in the action. The coefficient of equation (B.3) linear in $U_{X_{1}^{\prime} X_{1}}$ is then

$$
\begin{aligned}
\frac{1}{2} \frac{d}{d \tau} \tilde{T}_{\ell=0}\left(\tau X_{1} X_{1}^{\prime}\right) & =\frac{d}{d \tau}\left\langle H(\tau) \psi\left(X_{1}\right) \psi^{\dagger}\left(X_{1}^{\prime}\right)\right\rangle \\
& =\hbar \delta\left(\tau-\tau_{1}^{\prime}\right) \frac{\partial}{\partial \tau_{1}^{\prime}} G_{X_{1} X_{1}^{\prime}}+\hbar \delta\left(\tau-\tau_{1}\right) \frac{\partial}{\partial \tau_{1}} G_{X_{1} X_{1}^{\prime}} .
\end{aligned}
$$

A Fourier transform of the viscosity response function from imaginary time $\tau$ to Matsubara frequency $i \omega_{m}$ and from $X, X^{\prime}$ to incoming momenta $K_{1}=\left(\boldsymbol{k}, i \epsilon_{n}\right)$ and outgoing momenta $K_{1}^{\prime}=\left(\boldsymbol{k}, i \epsilon_{n}+i \omega_{m}\right)$ yields $\tilde{T}_{\ell=0}\left(i \omega_{m} K_{1}^{\prime} K_{1}\right)$, or

$$
\begin{aligned}
\frac{i \hbar \omega_{m}}{2} \tilde{T}_{\ell=0}\left(k, i \omega_{m},\right. & \left.i \epsilon_{n}+i \omega_{m}, i \epsilon_{n}\right) \\
& =\left(i \hbar \epsilon_{n}+i \hbar \omega_{m}+\mu\right) G\left(k, i \epsilon_{n}+i \omega_{m}\right)-\left(i \hbar \epsilon_{n}+\mu\right) G\left(k, i \epsilon_{n}\right) .
\end{aligned}
$$

Hence, the bulk viscosity response function is given by a difference of two single-particle Green's functions multiplied by their energies. This Ward identity holds exactly and has been derived without reference to the T-matrix approximation used above in section 5 . One can easily check the Ward identity in the non-interacting case where $G\left(k, i \epsilon_{n}\right)=$ $-\left(i \hbar \epsilon_{n}-\varepsilon_{k}+\mu\right)^{-1}$ and $\tilde{T}_{\ell=0}^{(0)}\left(k, i \omega_{m}, i \epsilon_{n}+i \omega_{m}, i \epsilon_{n}\right)=G\left(k, i \epsilon_{n}+i \omega_{m}\right) 2 \varepsilon_{k} G\left(k, i \epsilon_{n}\right)$. As an alternative to starting with equation (B.1), which is already a consequence of scale invariance, we have also derived the Ward identity by applying the local conformal transformation $\beta(t)$ in Ref. [30] to the generating functional of the Green's functions, with the same result. We note that scale plus Galilean invariance imply conformal invariance [82] and that both are necessary for a vanishing bulk viscosity [24].

A corresponding Ward identity holds for the bosonic viscosity response function (32), which is given by the difference of two vertex functions $\Gamma(K)=\Gamma\left(\boldsymbol{k}, i \Omega_{m}\right)$,

$$
\begin{aligned}
\frac{i \hbar \omega_{m}}{2} \tilde{S}_{\ell=0}\left(k, i \omega_{m}, i \Omega_{m}+i \omega_{m}, i \Omega_{m}\right) & \\
& =\left(i \hbar \Omega_{m}+i \hbar \omega_{m}+2 \mu\right) \Gamma\left(k, i \Omega_{m}\right)-\left(i \hbar \Omega_{m}+2 \mu\right) \Gamma\left(k, i \Omega_{m}+i \omega_{m}\right) .
\end{aligned}
$$

These Ward identities provide a solution to the T-matrix transport equations (38)-(43), as one can check by plugging in the Ward identities (B.5) and (B.6) on the right-hand side. Thus we have proven that the T-matrix approximation conserves not only charge, momentum and energy but also the dilatation current (scale invariance).

From the Ward identities of scale invariance it follows that the bulk viscosity computed via equation (30) vanishes for any frequency, temperature or density,

$$
\operatorname{Re} \zeta(\omega) \equiv 0 \text {. }
$$


Indeed, the fermionic Ward identity (B.5) for $\tilde{T}_{\ell}\left(K_{1}, K\right)$ implies by a shift of the frequency that the first term of the stress correlation function (30) vanishes identically. The bosonic Ward identity (B.6) implies, again by a shift of frequency, that

$$
\frac{1}{\beta} \sum_{i \Omega_{m}} \tilde{S}_{\ell=0}\left(k, i \omega_{m}, i \Omega_{m}+i \omega_{m}, i \Omega_{m}\right)=\frac{1}{\beta} \sum_{i \Omega_{m}} 4 \Gamma\left(k, i \Omega_{m}\right),
$$

such that also the second term of the correlation function (30) vanishes identically.

This result has been obtained previously in different ways [25, 24, 21]. For instance, it follows in a rather direct manner from a sum rule

$$
\frac{1}{\pi} \int_{0}^{\infty} d \omega \zeta(\omega)=\frac{1}{72 \pi m a^{2}}\left(\frac{\partial C}{\partial a^{-1}}\right)_{s}
$$

for the bulk viscosity that has been derived by Taylor and Randeria [28]. The fact that the derivative $\partial C / \partial(1 / a)$ is finite at unitarity and that $\zeta(\omega) \geq 0$ is a positive function, immediately implies that the bulk viscosity of the unitary gas at $a^{-1}=0$ vanishes at arbitrary frequencies.

\section{Appendix C. Relation between contact and tail coefficient}

One can compute the viscosity tail $C_{\eta}$ defined in equation (58),

$$
\eta(\omega \rightarrow \infty)=\frac{\hbar^{3 / 2} C_{\eta}}{\sqrt{m \omega}}
$$

in at least four different ways: from the current correlation function, from the stress tensor correlation function, from the relation between the shear viscosity and the density correlation function, and from the viscosity sum rule (13). In this Appendix we will analytically compute $C_{\eta}$ at unitarity by the first two methods, thus confirming equation (59). The identical result is obtained by considering the high-frequency behavior of the density correlation function, as has already been shown in section 7 , and from the sum rule.

The trace of the current correlation functions is in general a linear combination of shear and bulk viscosities [28]

$$
\begin{aligned}
\chi_{J}(\boldsymbol{q}, \omega) & \equiv\left\langle\left[j_{k}(\boldsymbol{q}, \omega), j_{k}(-\boldsymbol{q},-\omega)\right]\right\rangle \\
& =\chi_{L}(\boldsymbol{q}, \omega)+2 \chi_{T}(\boldsymbol{q}, \omega) \\
& =\frac{i q^{2}}{\omega}\left[\frac{10}{3} \eta(\omega)+\zeta(\omega)\right]+\mathcal{O}\left(q^{4}\right) .
\end{aligned}
$$

At unitarity $\zeta(\omega) \equiv 0$, and the real shear viscosity is given by

$$
\eta(\omega)=\frac{3}{10} \lim _{q \rightarrow 0} \frac{\omega}{q^{2}} \chi_{J}^{\prime \prime}(\boldsymbol{q}, \omega) .
$$

In the asymptotic high-frequency limit $\omega \rightarrow \infty$ only the diagrammatic contributions at first order in the pair propagator contribute to the tail. These are the self-energy 
(S), Maki-Thompson (MT) and Aslamazov-Larkin (AL) diagrams in Fig. 3 (see also [77, Fig. 6]),

$$
\chi_{J}(\boldsymbol{q}, \omega)=\chi_{J}^{\mathrm{S}}(\boldsymbol{q}, \omega)+\chi_{J}^{\mathrm{MT}}(\boldsymbol{q}, \omega)+\chi_{J}^{\mathrm{AL}}(\boldsymbol{q}, \omega) .
$$

In each of these diagrams the vertex function $\Gamma_{X X^{\prime}}$ is replaced by its value at $X^{\prime}=X^{+}$,

$$
-\tilde{g}^{2}\left\langle\bar{\psi}_{B} \psi_{B}\right\rangle=\left.\Gamma_{X X^{\prime}}\right|_{X^{\prime}=X^{+}}=\sum_{\boldsymbol{k}} \frac{1}{\beta} \sum_{i \Omega_{m}} \Gamma\left(\boldsymbol{k}, i \Omega_{m}\right)=-\frac{\hbar^{4} C}{m^{2}}
$$

which defines the contact density $C$ [29]. The remaining part of the diagram is evaluated at zero bosonic wavenumber $\boldsymbol{k}=0$ and Matsubara frequency $\Omega_{m}=0$,

$$
\Gamma\left(\boldsymbol{k}, i \Omega_{m}\right) \mapsto-\frac{\hbar^{4} C}{m^{2}} \delta(\boldsymbol{k}) \delta\left(\Omega_{m}\right) .
$$

To this order in the pair propagator, the fermionic Green's functions are bare $G_{0}\left(\boldsymbol{p}, i \epsilon_{n}\right)=$ $-\left(i \hbar \epsilon_{n}-\varepsilon_{\boldsymbol{p}}\right)^{-1}$ and have spectral weight only on-shell. Then to absorb an external high-energy perturbation with frequency $\omega$, fermions are excited to very high momenta $2 \varepsilon_{\boldsymbol{p}} \approx \hbar \omega$, which are much larger than the Fermi or thermal momentum scales. Hence only few-body physics is probed and it is sufficient to evaluate the diagrams in the vacuum limit $\mu=0$ and set the Fermi functions to zero.

Specifically, we obtain for the self-energy contribution to the current correlation function (C.3)

$$
\begin{aligned}
\chi_{J}^{\mathrm{S}}\left(\boldsymbol{q}, i \omega_{m}\right)= & \frac{\hbar^{4} C}{m^{2}} \sum_{\sigma} \sum_{\boldsymbol{p}} j_{k}(\boldsymbol{p}, \boldsymbol{p}+\boldsymbol{q}) j_{k}(\boldsymbol{p}+\boldsymbol{q}, \boldsymbol{p}) \\
& \times \frac{1}{\beta} \sum_{i \epsilon_{n}} G^{2}\left(\boldsymbol{p}, i \epsilon_{n}\right) G\left(-\boldsymbol{p},-i \epsilon_{n}\right) G\left(\boldsymbol{p}+\boldsymbol{q}, i \epsilon_{n}+i \omega_{m}\right)+\left(-\boldsymbol{q},-i \omega_{m}\right) \\
= & \frac{\hbar^{4} C}{m^{2}} 2 \sum_{\boldsymbol{p}} \frac{p^{2}}{4 \varepsilon_{\boldsymbol{p}_{-}}^{2}\left(i \hbar \omega_{m}-\varepsilon_{\boldsymbol{p}_{-}}-\varepsilon_{\boldsymbol{p}_{+}}\right)}+\left(-\boldsymbol{q},-i \omega_{m}\right)
\end{aligned}
$$

with the current vertex insertion $\boldsymbol{p}$ squared and shifted momenta $\boldsymbol{p}_{ \pm}=\boldsymbol{p} \pm \boldsymbol{q} / 2$. Likewise, the Maki-Thompson contribution is

$$
\begin{aligned}
\chi_{J}^{\mathrm{MT}}\left(\boldsymbol{q}, i \omega_{m}\right)= & \frac{\hbar^{4} C}{m^{2}} \sum_{\sigma} \sum_{\boldsymbol{p}} j_{k}(\boldsymbol{p}, \boldsymbol{p}+\boldsymbol{q}) j_{k}(\boldsymbol{p}+\boldsymbol{q}, \boldsymbol{p}) \\
& \times \frac{1}{\beta} \sum_{i \epsilon_{n}} G\left(\boldsymbol{p}, i \epsilon_{n}\right) G\left(-\boldsymbol{p},-i \epsilon_{n}\right) G\left(\boldsymbol{p}+\boldsymbol{q}, i \epsilon_{n}+i \omega_{m}\right) G\left(-\boldsymbol{p}-\boldsymbol{q},-i \epsilon_{n}-i \omega_{m}\right) \\
= & \frac{\hbar^{4} C}{m^{2}} 2 \sum_{\boldsymbol{p}} \frac{p^{2}}{4 \varepsilon_{\boldsymbol{p}_{-}} \varepsilon_{\boldsymbol{p}_{+}}}\left(\frac{1}{i \hbar \omega_{m}-\varepsilon_{\boldsymbol{p}_{-}}-\varepsilon_{\boldsymbol{p}_{+}}}+\frac{1}{-i \hbar \omega_{m}-\varepsilon_{\boldsymbol{p}_{-}}-\varepsilon_{\boldsymbol{p}_{+}}}\right) .
\end{aligned}
$$

Expanding to order $q^{2}$ and performing the integrals yields the retarded correlation function

$$
\chi_{J}^{\mathrm{S}}(\boldsymbol{q}, \omega+i 0)+\chi_{J}^{\mathrm{MT}}(\boldsymbol{q}, \omega+i 0)=\frac{(i-1) \hbar^{3 / 2} C q^{2}}{3 \pi m^{1 / 2} \omega^{3 / 2}}
$$


and the shear viscosity tail

$$
\eta_{\mathrm{S}+\mathrm{MT}}(\omega \rightarrow \infty)=\frac{\hbar^{3 / 2} C}{10 \pi \sqrt{m \omega}}
$$

with tail coefficient $C_{\eta}^{\mathrm{S}+\mathrm{MT}}=C /(10 \pi)$.

In the Aslamazov-Larkin contribution, either one of the two vertex functions is replaced by the contact and the other one is left in place, with the external frequency $\omega$ and momentum $\boldsymbol{q}$ as arguments:

$$
\chi_{J}^{\mathrm{AL}}\left(\boldsymbol{q}, i \omega_{m}\right)=-\frac{\hbar^{4} C}{m^{2}}\left[S_{k, \ell=1}\left(\boldsymbol{q}, i \omega_{m}\right)\right]^{2} \Gamma\left(\boldsymbol{q}, i \omega_{m}\right)+\left(-\boldsymbol{q},-i \omega_{m}\right) .
$$

The $\ell=1$ bosonic response vertex with one current vertex insertion $p_{k}$ is given by

$$
\begin{aligned}
S_{k, \ell=1}\left(\boldsymbol{q}, i \omega_{m}\right) & =-2 \sum_{\boldsymbol{p}} j_{k}(\boldsymbol{p}, \boldsymbol{p}+\boldsymbol{q}) \frac{1}{\beta} \sum_{i \epsilon_{n}} G\left(\boldsymbol{p}, i \epsilon_{n}\right) G\left(-\boldsymbol{p},-i \epsilon_{n}\right) G\left(\boldsymbol{p}+\boldsymbol{q}, i \epsilon_{n}+i \omega_{m}\right) \\
& =2 \sum_{\boldsymbol{p}} \frac{p_{k}}{2 \varepsilon_{\boldsymbol{p}_{-}}\left(i \hbar \omega_{m}-\varepsilon_{\boldsymbol{p}_{-}}-\varepsilon_{\boldsymbol{p}_{+}}\right)} .
\end{aligned}
$$

Integration and analytical continuation to a retarded function yields

$$
S_{k, \ell=1}(\boldsymbol{q}, \omega+i 0)=\frac{i m^{3 / 2} q_{k}}{6 \pi \sqrt{\hbar \omega}} .
$$

Since $\left[S_{k, \ell=1}(\boldsymbol{q}, \omega)\right]^{2}=-m^{3} q^{2} /\left(36 \pi^{2} \hbar \omega\right)$ is already of order $q^{2}$ it is sufficient to evaluate the vertex function (49) at zero momentum, $\Gamma^{\mathrm{ret}}(\boldsymbol{q}=0, \omega)=-4 \pi i \hbar^{5 / 2} m^{-3 / 2} \omega^{-1 / 2}$ to obtain the AL correlation function

$$
\chi_{J}^{\mathrm{AL}}(\boldsymbol{q}, \omega+i 0)=-i \frac{\hbar^{3 / 2} C q^{2}}{9 \pi m^{1 / 2} \omega^{3 / 2}}
$$

and the shear viscosity tail

$$
\eta_{\mathrm{AL}}(\omega \rightarrow \infty)=-\frac{\hbar^{3 / 2} C}{30 \pi \sqrt{m \omega}} .
$$

The resulting total tail is

$$
\eta(\omega \rightarrow \infty)=\frac{\hbar^{3 / 2} C}{15 \pi \sqrt{m \omega}}
$$

with tail coefficient $C_{\eta}=C /(15 \pi)$, in agreement with both the exact sum rule (13) and our numerical data.

An alternative way to compute the tail in the frequency dependent viscosities is to consider the stress tensor correlation functions in the limit $\omega \rightarrow \infty$. The calculation is analogous to the case of the current correlations, except that there are now stress vertex insertions, and one can let the external momentum $\boldsymbol{q} \rightarrow 0$. Note that the interaction 
contribution to the shear stress operator vanishes for the zero-range model of the unitary Fermi gas, cf. section 5. We obtain

$$
C_{\eta}^{\Pi, \mathrm{S}}=C_{\eta}^{\Pi, \mathrm{MT}}=\frac{C}{30 \pi}, \quad C_{\eta}^{\Pi, \mathrm{AL}}=0
$$

in agreement with equation (C.10). For the case of the bulk viscosity,

$$
C_{\zeta}^{\Pi, \mathrm{S}}=C_{\zeta}^{\Pi, \mathrm{MT}}=\frac{C}{18 \pi}, \quad \quad C_{\zeta}^{\Pi, \mathrm{AL}}=-\frac{C}{9 \pi}
$$

such that the total $C_{\zeta}=0$ vanishes in accordance with scale invariance (B.7).

\section{References}

[1] G. Policastro, D. T. Son, A. O. Starinets, Phys. Rev. Lett. 87 (2001) 081601.

[2] P. Kovtun, D. T. Son, A. O. Starinets, Phys. Rev. Lett. 94 (2005) 111601.

[3] T. Schaefer, D. Teaney, Rep. Prog. Phys. 72 (2009) 126001.

4] T. D. Cohen, Phys. Rev. Lett. 99 (2007) 021602

[5] D. T. Son, Phys. Rev. Lett. 100 (2008) 029101.

[6] M. Brigante, H. Liu, R. C. Myers, S. Shenker, S. Yaida, Phys. Rev. Lett. 100 (2008) 191601.

[7] Y. Kats, P. Petrov, J. High Energy Phys. 01 (2009) 044.

[8] A. Buchel, R. C. Myers, A. Sinha, J. High Energy Phys. 2009 (2009) 084.

[9] S. A. Hartnoll, Classical and Quantum Gravity 26 (2009) 224002.

[10] J. McGreevy, Holographic duality with a view toward many-body physics, 2009. Preprint arXiv:0909.0518.

[11] S. Sachdev, Condensed matter and AdS/CFT, 2010. Preprint arXiv:1002.2947.

[12] H. B. Meyer, Phys. Rev. D 76 (2007) 101701.

[13] H. B. Meyer, Phys. Rev. Lett. 100 (2008) 162001.

[14] H. B. Meyer, J. High Energy Phys. 2008 (2008) 031.

[15] S. Sachdev, Quantum Phase Transitions, Cambridge University Press, 1998.

[16] M. Müller, J. Schmalian, L. Fritz, Phys. Rev. Lett. 103 (2009) 025301.

[17] D. S. Novikov, Viscosity of a two-dimensional Fermi liquid, 2006. Preprint arXiv:cond-mat/0603184.

[18] W. Ketterle, M. Zwierlein, in: M. Inguscio, W. Ketterle, C. Salomon (Eds.), Ultracold Fermi Gases, Proceedings of the International School of Physics "Enrico Fermi", Varenna, IOS Press, Amsterdam, 2008.

[19] I. Bloch, J. Dalibard, W. Zwerger, Rev. Mod. Phys. 80 (2008) 885-964.

[20] S. Giorgini, L. Pitaevskii, S. Stringari, Rev. Mod. Phys. 80 (2008) 1215-1274.

[21] Y. Nishida, D. T. Son, Phys. Rev. D 76 (2007) 086004

[22] P. Nikolić, S. Sachdev, Phys. Rev. A 75 (2007) 033608.

[23] T. L. Ho, Phys. Rev. Lett. 92 (2004) 090402.

[24] D. T. Son, Phys. Rev. Lett. 98 (2007) 020604.

[25] F. Werner, Y. Castin, Phys. Rev. A 74 (2006) 053604.

[26] G. M. Bruun, H. Smith, Phys. Rev. A 75 (2007) 043612.

[27] G. Rupak, T. Schaefer, Phys. Rev. A 76 (2007) 053607.

[28] E. Taylor, M. Randeria, Phys. Rev. A 81 (2010) 053610.

[29] S. Tan, Ann. Physics 323 (2008) 2952-2970.

[30] D. T. Son, M. Wingate, Ann. Physics 321 (2006) 197-224.

[31] J. Wess, B. Zumino, Phys. Lett. B 37 (1971) 95-97.

[32] A. Zee, Quantum field theory in a nutshell, Princeton University Press, 2003.

[33] D. S. Petrov, Phys. Rev. A 67 (2003) 010703.

[34] Y. Nishida, D. T. Son, S. Tan, Phys. Rev. Lett. 100 (2008) 090405.

[35] Y. Castin, C. Mora, L. Pricoupenko, Four-body Efimov effect, 2010. Preprint arXiv:1006.4720.

[36] E. Braaten, H. W. Hammer, Phys. Rep. 428 (2006) 259-390.

[37] S. Moroz, R. Schmidt, Ann. Physics 325 (2009) 491-513.

[38] P. C. Martin, J. Schwinger, Phys. Rev. 115 (1959) 1342-1373.

[39] H. Smith, H. H. Jensen, Transport phenomena, Oxford University Press, USA, 1989. 
[40] J. P. Hansen, I. R. McDonald, Theory of simple liquids, Academic Press, 2006.

[41] P. C. Hohenberg, P. C. Martin, Ann. Physics 34 (1965) 291-359.

[42] D. Forster, Hydrodynamic fluctuations, broken symmetry, and correlation functions, WA Benjamin, 1975 .

[43] A. M. Polyakov, Zh. Eksp. Teor. Fiz. 30 (1969) 1164. [Zh. Eksp. Teor. Fiz. 57, 2144-2162 (1969)].

[44] S. Tan, Ann. Physics 323 (2008) 2971-2986.

[45] E. Braaten, L. Platter, Phys. Rev. Lett. 100 (2008) 205301.

[46] E. Braaten, D. Kang, L. Platter, Phys. Rev. A 78 (2008) 053606.

[47] R. Balian, From Microphysics to macrophysics, volume 2, Springer, 1992.

[48] L. D. Landau, I. M. Khalatnikov, Zh. Eksp. Teor. Fiz. 19 (1949) 637,709. English translation in Collected Papers of L. D. Landau (Pergamon Press, Ltd., Oxford, England, 1965), p. 494.

[49] R. B. Diener, R. Sensarma, M. Randeria, Phys. Rev. A 77 (2008) 023626.

[50] P. C. Hohenberg, B. I. Halperin, Rev. Mod. Phys. 49 (1977) 435-479.

[51] R. A. Ferrell, J. Low Temp. Phys. 70 (1988) 435-448.

[52] R. Biskeborn, R. W. Guernsey Jr, Phys. Rev. Lett. 34 (1975) 455-458.

[53] R. Schloms, J. Pankert, V. Dohm, Physica B 165-166 (1990) 563-564.

[54] G. Baym, L. P. Kadanoff, Phys. Rev. 124 (1961) 287-299.

[55] G. Baym, Phys. Rev. 127 (1962) 1391-1401.

[56] L. G. Aslamazov, A. I. Larkin, Phys. Lett. A 26 (1968) 238.

[57] S. Floerchinger, M. Scherer, S. Diehl, C. Wetterich, Phys. Rev. B 78 (2008) 174528.

[58] R. Haussmann, W. Rantner, S. Cerrito, W. Zwerger, Phys. Rev. A 75 (2007) 023610.

[59] R. Haussmann, M. Punk, W. Zwerger, Phys. Rev. A 80 (2009) 063612.

[60] R. Combescot, X. Leyronas, M. Y. Kagan, Phys. Rev. A 73 (2006) 023618.

[61] C. Cao, E. Elliott, J. Joseph, H. Wu, J. Petricka, T. Schaefer, J. E. Thomas, Observation of Universal Temperature Scaling in the Quantum Viscosity of a Unitary Fermi Gas, 2010. Preprint arXiv:1007.2625.

[62] A. Turlapov, J. Kinast, B. Clancy, L. Luo, J. Joseph, J. E. Thomas, J. Low Temp. Phys. 150 (2008) $567-576$.

[63] W. Götze, P. Wölfle, Phys. Rev. B 6 (1972) 1226-1238.

64] P. Massignan, G. M. Bruun, H. Smith, Phys. Rev. A 71 (2005) 033607.

[65] G. M. Bruun, H. Smith, Phys. Rev. A 72 (2005) 043605.

[66] T. L. Ho, E. J. Mueller, Phys. Rev. Lett. 92 (2004) 160404

[67] F. Werner, Y. Castin, Phys. Rev. Lett. 97 (2006) 150401.

[68] X. J. Liu, H. Hu, P. D. Drummond, Phys. Rev. Lett. 102 (2009) 160401.

[69] L. Luo, J. E. Thomas, J. Low Temp. Phys. 154 (2009) 1-29.

[70] S. Nascimbène, N. Navon, K. Jiang, F. Chevy, C. Salomon, Nature 463 (2010) 1057-1060.

[71] M. Horikoshi, S. Nakajima, M. Ueda, T. Mukaiyama, Science 327 (2010) 442.

[72] T. R. Kirkpatrick, D. Belitz, J. V. Sengers, J. Stat. Phys. 109 (2002) 373-405.

[73] G. M. Bruun, Few-Body Systems 45 (2009) 227-232.

[74] M. Zwierlein, 2010. Private communication; see also A. Sommer et al., abstract K1.3 at DAMOP 2010, Houston.

[75] M. Rangamani, S. F. Ross, D. T. Son, E. G. Thompson, J. High Energy Phys. 2009 (2009) 075.

[76] Z. Yu, G. M. Bruun, G. Baym, Phys. Rev. A 80 (2009) 23615.

[77] D. T. Son, E. G. Thompson, Phys. Rev. A 81 (2010) 063634.

[78] E. Burovski, E. Kozik, N. Prokof'ev, B. Svistunov, M. Troyer, Phys. Rev. Lett. 101 (2008) 090402.

[79] M. Punk, W. Zwerger, New J. Phys. 8 (2006) 168.

[80] D. Levesque, L. Verlet, J. Kürkijarvi, Phys. Rev. A 7 (1973) 1690-1700.

[81] S. Viscardy, J. Servantie, P. Gaspard, J. Chem. Phys. 126 (2007) 184512

[82] C. R. Hagen, Phys. Rev. D 5 (1972) 377-388. 